\title{
8 Fourier Series and Transforms
}

\subsection{Trigonometrical and Fourier Series}

Definition: A function $f(x)$ is said to be periodic if it is defined for all real $x$ and if there is some real number $T$ such that $f(x+T)=f(x)$. The number $T$ is then called the period of $f(x)$.

Examples: The sine and cosine functions have period $2 \pi$ and along with constants, which have arbitrary period, are the simplest periodic functions.

If it converges, a trigonometrical series of the form

$$
\frac{a_{0}}{2}+\sum_{n=1}^{\infty}\left(a_{n} \cos n x+b_{n} \sin n x\right)
$$

where $a_{0}, a_{1}, \ldots, a_{n}, \ldots, b_{1}, \ldots, b_{n}, \ldots$ are constants, has period $2 \pi$ also. The representation of a function $f(x)$ by such series was first investigated by Fourier in the context of heat conduction problems. Subsequently it was found that these series have an important role to play in the theory of functions of a real variable which is the reason for our interest in them.

Joseph Fourier (1768-1830) was a French mathematician and physicist whose early career advancement resulted as much from his active participation in the French Revolution and support of Napoleon Bonaparte as it did from the success of his research. Nevertheless, he is remembered for initiating the study of Fourier series and for discovering the law of conduction that is named after him. He is also generally acknowledged to have discovered the greenhouse effect.

Suppose that the series (8.1.1) converges uniformly to the function $f(x)$ :

$$
f(x)=\frac{a_{0}}{2}+\sum_{n=1}^{\infty}\left(a_{n} \cos n x+b_{n} \sin n x\right), \quad-\pi \leqslant x \leqslant \pi .
$$

We may multiply by $\cos m x$, where $m$ is a positive integer, and integrate term by term from $-\pi$ to $\pi$ to obtain

$$
\begin{aligned}
\int_{-\pi}^{\pi} f(x) \cos m x d x= & \frac{a_{0}}{2} \int_{-\pi}^{\pi} \cos m x d x \\
& +\sum_{n=1}^{\infty}\left[a_{n} \int_{-\pi}^{\pi} \cos n x \cos m x d x+b_{n} \int_{-\pi}^{\pi} \sin n x \cos m x d x\right] .
\end{aligned}
$$

Since $\cos n x \cos m x=\frac{1}{2} \cos (n+m) x+\frac{1}{2} \cos (n-m) x$ and $\sin n x \cos m x=\frac{1}{2} \sin (n+$ $m) x+\frac{1}{2} \sin (n-m) x$, we have the orthogonality relations

$$
\int_{-\pi}^{\pi} \cos n x \cos m x d x=\left\{\begin{array}{l}
0 \text { for } n \neq m \\
\pi \text { for } n=m
\end{array}\right.
$$




$$
\begin{gathered}
\int_{-\pi}^{\pi} \sin n x \cos m x d x=0 \text { for all } m, n \\
\int_{-\pi}^{\pi} \cos m x d x=\left\{\begin{array}{c}
0 \text { for } m \neq 0 \\
2 \pi \text { for } m=0
\end{array}\right.
\end{gathered}
$$

Applying these, we find

$$
\begin{aligned}
& a_{0}=\frac{1}{\pi} \int_{-\pi}^{\pi} f(x) d x \text { and } \\
& a_{m}=\frac{1}{\pi} \int_{-\pi}^{\pi} f(x) \cos m x d x, \quad m=1,2, \ldots
\end{aligned}
$$

Similarly, multiplying (8.1.2) by $\sin m x$, integrating term by term and using the orthogonality relation

$$
\int_{-\pi}^{\pi} \sin n x \sin m x d x=\left\{\begin{array}{l}
0 \text { for } n \neq m \\
\pi \text { for } n=m
\end{array},\right.
$$

we find

$$
b_{m}=\frac{1}{\pi} \int_{-\pi}^{\pi} f(x) \sin m x d x, \quad m=1,2, \ldots
$$

Equations (8.1.3) and (8.1.4) are called the Euler formulae for the coefficients and the set of numbers $\left\{a_{m}, b_{m}\right\}$ which they determine are called the Fourier coefficients of $f(x)$.

There is no a priori reason for supposing that a given function can be expanded in a uniformly convergent trigonometrical series. Therefore, the process we have just carried out is not a proof that the coefficients of a trigonometrical series representation of $f(x)$ are necessarily those determined by the Euler formulae. So, instead of starting with the series and the presumption that it has a certain property, we start from the function, calculate its Fourier coefficients, and determine the properties of the series that can be formed with them.

Definition: If we are given a function $f(x)$ that is integrable over the interval $-\pi \leqslant x \leqslant$ $\pi$, then the integrals in (8.1.3) and (8.1.4) that define its Fourier coefficients $\left\{a_{m}, b_{m}\right\}$ exist. The trigonometrical series of the form (8.1.1) that is constructed using these coefficients is called the Fourier series of $f(x)$.

The question which must be addressed now is whether the Fourier series of an arbitrary function $f(x)$ converges and if it does, whether its sum is $f(x)$. Because the sum of the series, if it exists, has period $2 \pi$, we use only the values that $f(x)$ assumes 
in the interval $-\pi \leqslant x \leqslant \pi$; outside this interval we define it by means of periodicity, $f(x \pm 2 \pi)=f(x)$, which gives us the periodic extension of $f(x)$.

Examples: Consider the function $x^{2}$. Its Fourier coefficients are

$$
\begin{aligned}
& a_{0}=\frac{1}{\pi} \int_{-\pi}^{\pi} x^{2} d x=\frac{2}{3} \pi^{2} \\
& a_{n}=\frac{1}{\pi} \int_{-\pi}^{\pi} x^{2} \cos n x d x=(-1)^{n} \frac{4}{n^{2}}, \quad n>0 \\
& b_{n}=\frac{1}{\pi} \int_{-\pi}^{\pi} x^{2} \sin n x d x=0 .
\end{aligned}
$$

Note that the sine coefficients are all zero because $x^{2}$ is an even function of $x$; similarly, all cosine coefficients vanish for odd functions of $x$.

The corresponding Fourier series

$$
f(x)=\frac{\pi^{2}}{3}+\sum_{n=1}^{\infty}(-1)^{n} \frac{4}{n^{2}} \cos n x
$$

is easily shown to be uniformly convergent for all values of $x$. As shown in Figure 8.1, its sum function, $f(x)$, reproduces the values assumed by $x^{2}$ on $-\pi \leqslant x \leqslant \pi$ and by its periodic extension outside that interval.

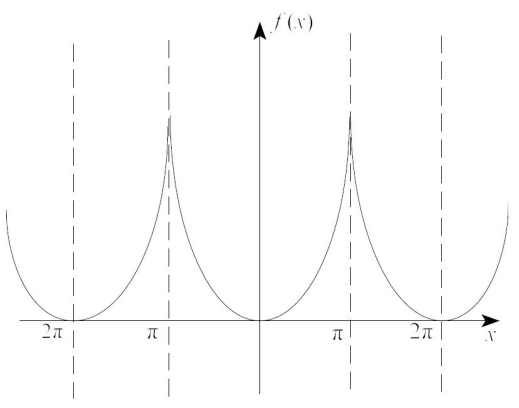

Figure 8.1: The Fourier series representation of $x^{2}$.

Next we consider the discontinuous function

$$
f(x)= \begin{cases}-1, & x<0 \\ +1, & x \geqslant 0\end{cases}
$$


This is an odd function so there are no cosine terms in its Fourier series, $a_{n}=0, n \geqslant 0$. As for the sine terms, the coefficients are

$$
b_{n}=\frac{2}{\pi} \int_{-\pi}^{\pi} \sin n x d x= \begin{cases}\frac{4}{n \pi}, & n=\text { odd } \\ 0, & n=\text { even }\end{cases}
$$

The corresponding series

$$
\frac{4}{\pi} \sum_{n=o d d}^{\infty} \frac{1}{n} \sin n x
$$

converges to

$-\quad+1$ for $0<x<\pi$,

$-\quad-1$ for $-\pi<x<0$, and

- $\quad 0$ for $x=-\pi, x=0$, and $x=+\pi$.

In other words, the Fourier series representation of $f(x)$ reproduces it throughout the interval except at the point of discontinuity, $x=0$, and at the end-points of the interval which are points of discontinuity for the periodic extension of $f(x)$. At these three exceptional points, the series converges to the mean of the right- and left-hand limits of (the periodic extension of) $f(x)$. This behaviour at the points of discontinuity is a general feature of Fourier series.

The points of discontinuity also result in the convergence being non-uniform in any interval that includes one of them. This is exhibited in Figure 8.2 which shows successive partial sums. Notice the overshoot on either side of the points of discontinuity. This too is a general feature of Fourier series and is called Gibbs' phenomenon. The overshoot remains finite as more and more terms are added and tends to the value 0.18 in the limit of infinitely many terms.

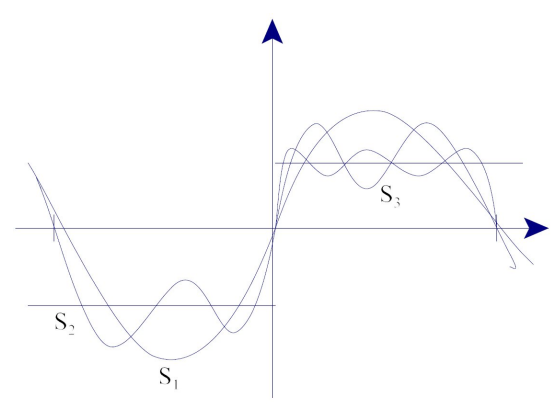

Figure 8.2: The first three partial sums of the Fourier series representation of a step function.

Finally, it is interesting to note that if we set $x=\pi / 2$ in the Fourier series, we have

$$
f\left(\frac{\pi}{2}\right)=1=\frac{4}{\pi}\left(1-\frac{1}{3}+\frac{1}{5}-+\ldots\right)
$$


or,

$$
\frac{\pi}{4}=1-\frac{1}{3}+\frac{1}{5}-\frac{1}{7}+-\ldots=\sum_{m=0} \frac{(-1)^{m}}{2 m+1}
$$

which is a series summation derived originally by Leibnitz using a geometrical argument.

\subsection{The Convergence Question}

There is a formal connection between Fourier series and Laurent series. Suppose that $f(z)$ is a single-valued analytic function, holomorphic in the annulus $R_{1}<|z|<R_{2}$. Then

$$
f(z)=\sum_{n=-\infty}^{\infty} c_{n} z^{n}
$$

where

$$
c_{n}=\frac{1}{2 \pi i} \int_{C} \frac{f(\zeta)}{\zeta^{n+1}} d \zeta
$$

and we can choose $C$ to be the circle $|\zeta|=r, R_{1}<r<R_{2}$.

Putting $z=r e^{i \theta}$, we have

$$
f\left(r e^{i \theta}\right)=\sum_{n=-\infty}^{\infty} A_{n} e^{i n \theta}
$$

where

$$
A_{n}=\frac{1}{2 \pi} \int_{-\pi}^{\pi} f\left(r e^{i \varphi}\right) e^{-i n \varphi} d \varphi .
$$

Combining terms pair wise for each value of $|n|$, we can rewrite the Laurent expansion as the Fourier series

$$
f\left(r e^{i \theta}\right)=A_{0}+\sum_{n=1}^{\infty}\left\{\left(A_{n}+A_{-n}\right) \cos n \theta+i\left(A_{n}-A_{-n}\right) \sin n \theta\right\},
$$

where

$$
\begin{gathered}
A_{0}=\frac{1}{2 \pi} \int_{-\pi}^{\pi} f\left(r e^{i \varphi}\right) d \varphi, \quad A_{n}+A_{-n}=\frac{1}{\pi} \int_{-\pi}^{\pi} f\left(r e^{i \varphi}\right) \cos n \varphi d \varphi, \\
i\left(A_{n}-A_{-n}\right)=\frac{1}{\pi} \int_{-\pi}^{\pi} f\left(r e^{i \varphi}\right) \sin n \varphi d \varphi .
\end{gathered}
$$

It follows from Laurent's Theorem that such a Fourier series converges uniformly to the function it represents. However, in general, one wants to represent a much larger class 
of functions than would meet the requirements of Laurent's Theorem. To see just how large a class admits such representation, we need to introduce some new concepts. The first of these is the concept of bounded variation.

Definition: Let $f(x)$ be defined on $a \leqslant x \leqslant b$ and let $x_{1}, x_{2}, \ldots, x_{n}$, be a set of points on that interval such that $a \leqslant x_{1} \leqslant x_{2} \leqslant \ldots \leqslant x_{n} \leqslant b$, as shown in Figure 8.3. Then, the sum

$$
\left|f(a)-f\left(x_{1}\right)\right|+\left|f\left(x_{1}\right)-f\left(x_{2}\right)\right|+\ldots+\left|f\left(x_{n}\right)-f(b)\right|
$$

is called the variation of $f(x)$ on the interval $a \leqslant x \leqslant b$ for the set of subdivisions $x_{1}, \ldots, x_{n}$. If the variation has an upper bound, $M$, independent of $n$, for all choices of $x_{1}, x_{2}, \ldots, x_{n}$, then $f(x)$ is said to be of bounded variation on $a \leqslant x \leqslant b$.

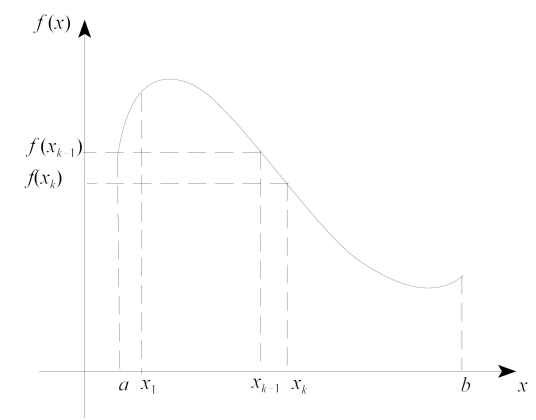

Figure 8.3: A set of points is introduced on the interval $a \leqslant x \leqslant b$ to define a corresponding variation of the function $f(x)$.

Examples: Two functions which are not of bounded variation are $f(x)=\frac{1}{x}$ and $f(x)=$ $\sin \frac{\pi}{x}$ on any interval that encloses $x=0$; see Figure 8.4. Examples of functions which are of bounded variation include piecewise continuous functions with a finite number of maxima and minima. A function $f(x)$ is piecewise continuous on a finite interval $a \leqslant x \leqslant b$ if the interval can be divided into finitely many sub-intervals, in each of which $f(x)$ is continuous and has finite limits as $x$ approaches either endpoint of the sub-interval from the interior.

We can now state without proof the theorem that establishes the conditions for point by point convergence of a Fourier series.

Fourier's Theorem: Let $f(x)$ be defined arbitrarily on $-\pi \leqslant x \leqslant \pi$ and defined for all other $x$ by its periodic extension, $f(x+2 \pi)=f(x)$. Also let $f(x)$ be such that $\int_{-\pi}^{\pi} f(x) d x$ exists, and if this be an improper integral, let it be absolutely convergent. Then, if $x$ is an interior point of any interval $a \leqslant x \leqslant b$ in which $f(x)$ is of bounded variation, the Fourier series of $f(x)$ converges at $x$ to the sum $\frac{1}{2}[f(x+0)+f(x-0)]$. Moreover, in every closed subinterval in which $f(x)$ is continuous, the convergence is uniform. 


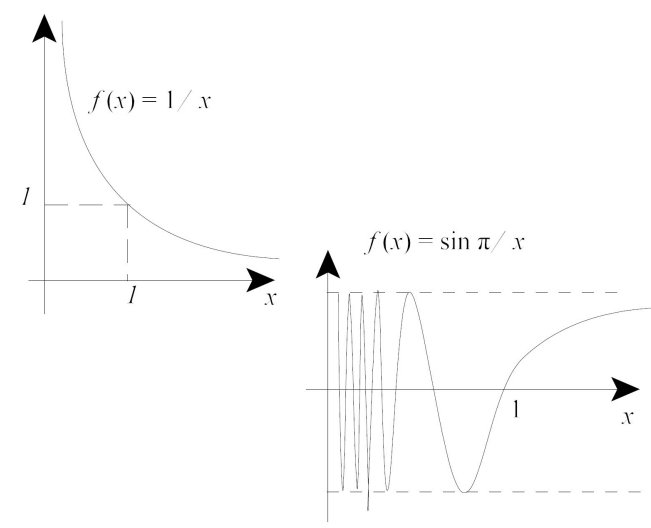

Figure 8.4: Two functions which are not of bounded variation in any interval containing $x=0$.

A proof of the theorem can be found in E. C. Titchmarsh, Theory of Functions, Oxford University Press, New York, 1964.

As we noted with the example in the preceding Section, whenever $f(x)$ has a finite or step discontinuity the series will converge to the mean of its values on either side of the point. In particular, if $a \leqslant-\pi$ and $b \geqslant \pi$, the Fourier series will converge to $\frac{1}{2}[f(-\pi+0)+f(\pi-0)]$ at $x= \pm \pi$; thus, the series will not reproduce $f(x)$ at these points unless $f(-\pi)=f(\pi)$.

While not terribly stringent, the conditions imposed on $f(x)$ in this theorem can be relaxed even more if we replace the requirement of pointwise convergence by that of convergence in the mean which is a form of convergence that is perfectly adequate for most physical applications. To introduce the concept, however, we need an additional definition.

Definition: The integral

$$
\int_{a}^{b}[f(x)-g(x)]^{2} d x
$$

for two functions $f(x)$ and $g(x)$ defined and square integrable on $a \leqslant x \leqslant b$ is called the square deviation of $f(x)$ and $g(x)$.

Evidently, a square deviation is a measure of how well one function "fits" another over the interval in question. We shall use it to meet our need for a more subtle form of convergence.

Definition: A sequence of functions $\left\{f_{n}(x)\right\}$ is said to converge in the mean to a function $f(x)$ on an interval $a \leqslant x \leqslant b$ if the corresponding square deviation of $f(x)$ and $f_{n}(x)$ tends to zero as $n \rightarrow \infty$ :

$$
\lim _{n \rightarrow \infty} \int_{a}^{b}\left[f(x)-f_{n}(x)\right]^{2} d x=0 .
$$


Convergence in the mean does not imply that $\lim _{n \rightarrow \infty} f_{n}(x)=f(x)$ at each point of $a \leqslant x \leqslant$ $b$. On the contrary, the limit of the sequence of functions may differ widely from $f(x)$ on a discrete set of points distributed over the integration interval and still produce a zero square deviation.

We shall now make use of square deviation and convergence in the mean to further our understanding of the representations of functions that are offered by trigonometrical series. Suppose that we have a square integrable function $f(x)$ defined on $-\pi \leqslant x \leqslant \pi$ and that we want to approximate it by the partial sum

$$
S_{n}(x)=\frac{A_{0}}{2}+\sum_{k=1}^{n}\left(A_{k} \cos k x+B_{k} \sin k x\right)
$$

where the coefficients $A_{k}, k=0,1,2, \ldots, n$, and $B_{k}, k=1,2, \ldots, n$, can be adjusted to achieve an optimal fit. In fact, we shall now try to determine what set of values for $\left\{A_{k}, B_{k}\right\}$ will minimize the square deviation

$$
D_{n}=\int_{-\pi}^{\pi}\left[f(x)-S_{n}(x)\right]^{2} d x
$$

Straightforward application of the orthogonality of the functions $\{\cos k x, \sin k x\}$ yields

$$
\begin{aligned}
D_{n}= & \int_{-\pi}^{\pi}[f(x)]^{2} d x+\left\{\frac{\pi}{2} A_{0}^{2}-A_{0} \int_{-\pi}^{\pi} f(x) d x\right\} \\
& +\sum_{k=1}^{n}\left\{\pi A_{k}^{2}-2 A_{k} \int_{-\pi}^{\pi} f(x) \cos k x d x\right\} \\
& +\sum_{k=1}^{n}\left\{\pi B_{k}^{2}-2 B_{k} \int_{-\pi}^{\pi} f(x) \sin k x d x\right\} .
\end{aligned}
$$

Using the Euler formulas for the Fourier coefficients of $f(x)$, we can rewrite this as

$$
D_{n}=\int_{-\pi}^{\pi}[f(x)]^{2} d x+\frac{\pi}{2}\left\{A_{0}^{2}-2 A_{0} a_{0}\right\}+\pi \sum_{k=1}^{n}\left\{A_{k}^{2}-2 A_{k} a_{k}+B_{k}^{2}-2 B_{k} b_{k}\right\} .
$$

But,

$$
A_{k}^{2}-2 A_{k} a_{k}=\left(A_{k}-a_{k}\right)^{2}-a_{k}^{2} \text { and } B_{k}^{2}-2 B_{k} b_{k}=\left(B_{k}-b_{k}\right)^{2}-b_{k}^{2}
$$

Thus,

$$
D_{n}=\int_{-\pi}^{\pi}[f(x)]^{2} d x-\pi\left\{\frac{1}{2} a_{0}^{2}+\sum_{k=1}^{n}\left[a_{k}^{2}+b_{k}^{2}\right]\right\}
$$




$$
+\pi\left\{\frac{1}{2}\left(A_{0}-a_{0}\right)^{2}+\sum_{k=1}^{n}\left[\left(A_{k}-a_{k}\right)^{2}+\left(B_{k}-b_{k}\right)^{2}\right]\right\} .
$$

The last line in this expression is positive-definite:

$$
\frac{1}{2}\left(A_{0}-a_{0}\right)^{2}+\sum_{k=1}^{n}\left[\left(A_{k}-a_{k}\right)^{2}+\left(B_{k}-b_{k}\right)^{2}\right] \geqslant 0 .
$$

Therefore, the square deviation is minimized if the trigonometrical series coefficients $A_{k}$ and $B_{k}$ are chosen to be the Fourier coefficients of $f(x)$ :

$$
\left[D_{n}\right]_{\min }=\int_{-\pi}^{\pi}[f(x)]^{2} d x-\pi\left\{\frac{1}{2} a_{0}^{2}+\sum_{k=1}^{n}\left[a_{k}^{2}+b_{k}^{2}\right]\right\} .
$$

Since $D_{n} \geqslant 0$, we have

$$
\frac{a_{0}^{2}}{2}+\sum_{k=1}^{n}\left[a_{k}^{2}+b_{k}^{2}\right] \leqslant \frac{1}{\pi} \int_{-\pi}^{\pi}[f(x)]^{2} d x .
$$

This holds for any $n$ and as $n$ increases, the sequence on the left is monotonically increasing but bounded by the integral on the right. Therefore, it possesses a limit as $n \rightarrow \infty$ and the limit satisfies

$$
\frac{a_{0}^{2}}{2}+\sum_{k=1}^{\infty}\left[a_{k}^{2}+b_{k}^{2}\right] \leqslant \frac{1}{\pi} \int_{-\pi}^{\pi}[f(x)]^{2} d x
$$

which is known as Bessel's inequality.

Suppose that we now require that the square deviation tend to zero as $n \rightarrow \infty$ :

$$
\lim _{n \rightarrow \infty}\left[D_{n}\right]_{\min }=0 \text {. }
$$

By definition, this would mean that the Fourier series

$$
\frac{a_{0}}{2}+\sum_{n=0}^{\infty}\left[a_{n} \cos n x+b_{n} \sin n x\right]
$$

converges in the mean to $f(x)$. It also means that the above inequality becomes the equality

$$
\frac{a_{0}^{2}}{2}+\sum_{k=1}^{\infty}\left[a_{k}^{2}+b_{k}^{2}\right]=\frac{1}{\pi} \int_{-\pi}^{\pi}[f(x)]^{2} d x
$$

which is known as Parseval's equation.

Whenever Parseval's equation holds for a certain class of functions $f(x)$, we say that the set of trigonometrical functions $\{\cos n x, \sin n x\}$ is complete with respect to 
that class and Parseval's equation is called the completeness relation. The question that we now want to answer is what is the largest class of functions for which completeness has been established and hence, for which convergence in the mean of their Fourier series is assured. The formal answer, known as the Riesz-Fischer Theorem, makes use of a form of integration that differs somewhat from Riemann integration. Called Lebesgue integration, it is defined for all bounded functions, including those that may be discontinuous on an infinite but enumerable set of points in the interval of integration, and even for some unbounded functions. Thus, the class of Lebesgue integrable functions is much larger than the class of functions which are of bounded variation or which are Riemann integrable. Nevertheless, when both the Lebesgue and the Riemann integrals of a function exist, they yield identical results. Moreover, when two functions are equal almost everywhere, that is to say everywhere except on an enumerable set of points, they have the same Lebesgue integral. Therefore, in practice, we can usually proceed by using Riemann integration techniques without regard to discontinuous or even, in some circumstances, unbounded behaviour provided that it is restricted to an enumerable point set.

Theorem: The set of trigonometrical functions $\{\cos n x, \sin n x\}$ is complete with respect to those functions $f(x)$ that are (Lebesgue) square integrable on the interval $-\pi \leqslant x \leqslant \pi$ and hence satisfy

$$
\int_{-\infty}^{\infty}[f(x)]^{2} d x<\infty
$$

Thus, the Fourier coefficients $\left\{a_{n}, b_{n}\right\}$ for all such functions satisfy Parseval's equation and the corresponding Fourier series converge in the mean to the functions they represent.

This completes our discussion of the "convergence question".

\subsection{Functions Having Arbitrary Period}

Periodic functions in applications rarely have period $2 \pi$ but the transition from period $2 \pi$ to any period $T$ can be effected by a simple change of scale. Suppose that $f(t)$ has period $T$. Then we can introduce a new variable $x$ such that $f(t)$, as a function of $x$, has period $2 \pi$ by setting $x=\frac{2 \pi}{T} t$. Hence, if $f(t)$ has a Fourier series, it must be of the form

$$
f(t)=f\left(\frac{T}{2 \pi}\right)=\frac{a_{0}}{2}+\sum_{n=1}^{\infty}\left(a_{n} \cos n x+b_{n} \sin n x\right),
$$

with coefficients given by the Euler formulas:

$$
a_{0}=\frac{1}{2 \pi} \int_{-\pi}^{\pi} f\left(\frac{T}{2 \pi}\right) d x, \quad a_{n}=\frac{1}{2 \pi} \int_{-\pi}^{\pi} f\left(\frac{T}{2 \pi}\right) \cos n x d x,
$$




$$
b_{n}=\frac{1}{\pi} \int_{-\pi}^{\pi} f\left(\frac{T}{2 \pi}\right) \sin n x d x .
$$

Since $x=\frac{2 \pi}{T}$, we have $d x=\frac{2 \pi}{T} d t$ and the interval of integration becomes

$$
-\frac{T}{2} \leqslant t \leqslant \frac{T}{2}
$$

Consequently, the Fourier coefficients of the periodic function $f(t)$ of period $T$ must be given by

$$
\begin{aligned}
& a_{0}=\frac{2}{T} \int_{-T / 2}^{T / 2} f(t) d t \\
& a_{n}=\frac{2}{T} \int_{-T / 2}^{T / 2} f(t) \cos \frac{2 n \pi t}{T} d t \\
& b_{n}=\frac{2}{T} \int_{-T / 2}^{T / 2} f(t) \sin \frac{2 n \pi t}{T} d t, \quad n=1,2,3, \ldots
\end{aligned}
$$

Furthermore, the Fourier series (8.3.1) with $x$ expressed in terms of $t$ becomes

$$
f(t)=\frac{a_{0}}{2}+\sum_{n=1}^{\infty}\left(a_{n} \cos \frac{2 n \pi t}{T}+b_{n} \sin \frac{2 n \pi t}{T}\right) .
$$

The interval of integration in (8.3.2) may be replaced by any interval of length $T$ : $0 \leqslant t \leqslant T$, for example.

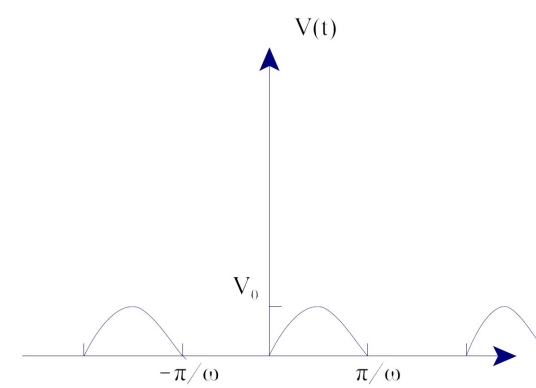

Figure 8.5: Half-Wave Rectifier

Example: Suppose that we wish to Fourier analyze the outcome of passing a sinusoidal voltage $V_{0} \sin \omega t$ through a half-wave rectifier that clips the negative portion of 
the wave. The rectified potential will continue to have period $T=\frac{2 \pi}{\omega}$ but will have the new functional form (see Figure 8.5)

$$
V(t)=\left\{\begin{array}{cc}
0 & \text { for }-T / 2<t<0, \\
V_{0} \sin \omega t & \text { for } 0<t<T / 2
\end{array}\right.
$$

Since $V=0$ when $-T / 2<t<0$, we obtain from (8.3.2)

$$
a_{0}=\frac{\omega}{\pi} \int_{0}^{\pi / \omega} V_{0} \sin \omega t d t=\frac{2 V_{0}}{\pi}
$$

and

$$
a_{n}=\frac{\omega}{\pi} \int_{0}^{\pi / \omega} V_{0} \sin \omega t \cos n \omega t d t=\frac{\omega V_{0}}{2 \pi} \int_{0}^{\pi / \omega}[\sin (1+n) \omega t+\sin (1-n) \omega t] d t .
$$

When $n=1$, the integral on the right is zero, and when $n=2,3, \ldots$, we obtain

$$
\begin{aligned}
a_{n} & =\frac{\omega V_{0}}{2 \pi}\left[-\frac{\cos (1+n) \omega t}{(1+n) \omega}-\frac{\cos (1-n) \omega t}{(1-n) \omega}\right]_{0}^{\pi / \omega} \\
& =\frac{V_{0}}{2 \pi}\left(\frac{1-\cos (1+n) \pi}{1+n}+\frac{1-\cos (1-n) \pi}{1-n}\right) .
\end{aligned}
$$

When $n$ is odd, this is zero; for even $n$ it gives

$$
a_{n}=\frac{V_{0}}{2 \pi}\left(\frac{2}{1+n}+\frac{2}{1-n}\right)=-\frac{2 V_{0}}{(n-1)(n+1) \pi}, \quad n=2,4, \ldots .
$$

In a similar fashion we find that $b_{1}=V_{0} / 2$ and $b_{n}=0$ for $n=2,3, \ldots$ Consequently,

$$
V(t)=\frac{V_{0}}{\pi}+\frac{V_{0}}{2} \sin \omega t-\frac{2 V_{0}}{\pi}\left(\frac{1}{1 \cdot 3} \cos 2 \omega t+\frac{1}{3 \cdot 5} \cos 4 \omega t+\ldots\right) .
$$

In many applications the independent variable is distance $x$ and the most intuitive way to denote the period is to use $2 L$. In that case the formulas read

$$
f(x)=\frac{a_{0}}{2}+\sum_{n=1}^{\infty}\left[a_{n} \cos \frac{n \pi x}{L}+b_{n} \sin \frac{n \pi x}{L}\right],
$$

with

$$
a_{n}=\frac{1}{L} \int_{-L}^{L} f(x) \cos \frac{n \pi x}{L} d x, \quad b_{n}=\frac{1}{L} \int_{-L}^{L} f(x) \sin \frac{n \pi x}{L} d x
$$




\subsection{Half-Range Expansions - Fourier Sine and Cosine Series}

We have already seen that certain types of functions have particularly simple Fourier series. If $f(t)$ is an even function, $f(-t)=f(t)$, of period $T$ then its Fourier sine coefficients $b_{n}=0$ for all $n \geqslant 1$ and its Fourier series is a Fourier cosine series

$$
f(t)=\frac{a_{0}}{2}+\sum_{n=1}^{\infty} a_{n} \cos \frac{2 n \pi t}{T}
$$

with coefficients

$$
a_{0}=\frac{4}{T} \int_{0}^{T / 2} f(t) d t, \quad a_{n}=\frac{4}{T} \int_{0}^{T / 2} f(t) \cos \frac{2 n \pi t}{T} d t, \quad n=1,2,3, \ldots
$$

On the other hand, if $f(t)$ is odd, $f(-t)=-f(t)$, its Fourier cosine coefficients $a_{n}=0$ for all $n$ and its Fourier series is a Fourier sine series,

$$
f(t)=\sum_{n=1}^{\infty} b_{n} \sin \frac{2 n \pi t}{T}
$$

with coefficients

$$
b_{n}=\frac{4}{T} \int_{0}^{T / 2} f(t) \sin \frac{2 n \pi t}{T} d t .
$$

Suppose that we have a function $f(t)$ that is defined only on some finite interval $0 \leqslant t \leqslant \tau$. If we want to represent it by a Fourier series, we now have three distinct representations to choose from:

- $\quad$ a Fourier cosine series of period $T=2 \tau$,

- a Fourier sine series of period $T=2 \tau$, or

- a full Fourier series of period $T=\tau$.

As before, the latter series will converge to the periodic extension of $f(t)$. The first two, however, are half-range expansions that converge to periodic extensions of the functions $f_{1}(t)$ and $f_{2}(t)$ respectively, where

$$
f_{1}(t)=\left\{\begin{array}{cl}
f(t), & 0 \leqslant t \leqslant \tau \\
f(-t), & -\tau \leqslant t \leqslant 0
\end{array}\right.
$$

and

$$
f_{2}(t)=\left\{\begin{array}{c}
f(t), \quad 0<t \leqslant \tau \\
-f(-t), \quad-\tau \leqslant t<0
\end{array}\right.
$$

The first of these is called the symmetric extension and the second, the antisymmetric extension of $f(t)$. Thus, as shown in Figure 8.6, the Fourier cosine series

$$
f(t)=\frac{a_{0}}{2}+\sum_{n=1}^{\infty} a_{n} \cos \frac{n \pi t}{\tau}, \quad 0 \leqslant t \leqslant \tau
$$


with coefficients

$$
a_{n}=\frac{2}{\tau} \int_{0}^{\tau} f(t) \cos \frac{n \pi t}{\tau} d t, \quad n=0,1,2, \ldots,
$$

converges to the periodic symmetric extension of $f(t)$ of period $T=2 \tau$, while the Fourier sine series

$$
f(t)=\sum_{n=1}^{\infty} b_{n} \sin \frac{n \pi t}{\tau}, \quad 0<t<\tau
$$

with coefficients

$$
b_{n}=\frac{2}{\tau} \int_{0}^{\tau} f(t) \sin \frac{n \pi t}{\tau} d t, \quad n=1,2, \ldots,
$$

converges to the periodic antisymmetric extension of $f(t)$ of period $T=2 \tau$. The series (8.4.5) and (8.4.7) are called half-range expansions of $f(t)$.

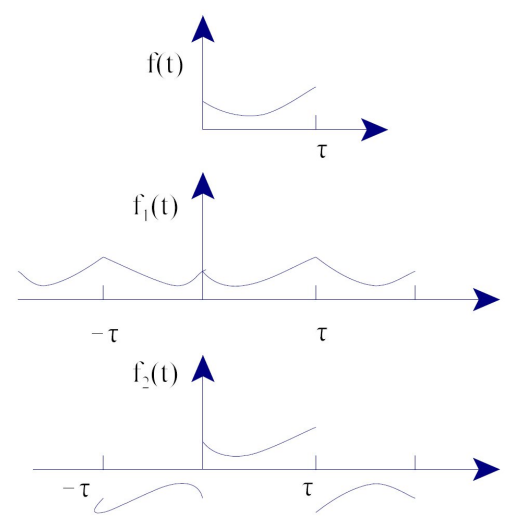

Figure 8.6: Periodic Extensions

Example: Consider the function $f(t)=\frac{t}{2 \tau}+\frac{1}{2}$. Its full-range Fourier coefficients are

$$
\begin{gathered}
a_{0}=1, a_{n}=\frac{1}{\tau} \int_{-\tau}^{\tau}\left(\frac{t}{2 \tau}+\frac{1}{2}\right) \cos \frac{n \pi t}{\tau} d t=0, \\
b_{n}=\frac{1}{\tau} \int_{-\tau}^{\tau}\left(\frac{t}{2 \tau}+\frac{1}{2}\right) \sin \frac{n \pi t}{\tau} d t=\frac{(-1)^{n+1}}{n \pi},
\end{gathered}
$$

and the Fourier series reads

$$
f(t)=\frac{1}{2}+\sum_{n=1}^{\infty} \frac{(-1)^{n+1}}{n \pi} \sin \frac{n \pi t}{\tau}, \quad-\tau<t<\tau .
$$



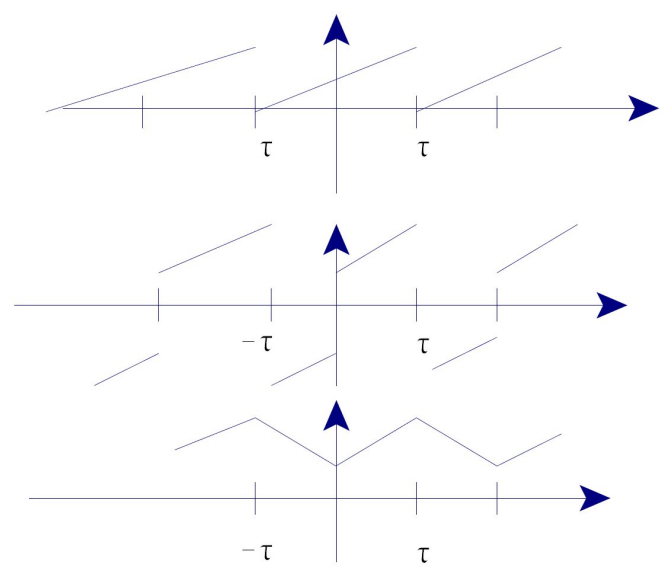

Figure 8.7: The Fourier, Fourier Sine and Fourier Cosine Series for $f(t)=\frac{t}{2 \tau}+\frac{1}{2}$.

The function's half-range Fourier sine coefficients are

$$
b_{n}=\frac{2}{\tau} \int_{0}^{\tau}\left(\frac{t}{2 \tau}+\frac{1}{2}\right) \sin \frac{n \pi t}{\tau} d t=\frac{1-2 \cos n \pi}{n \pi},
$$

and its Fourier sine series reads

$$
f(t)=\sum_{n=1}^{\infty} \frac{1-2(-1)^{n}}{n \pi} \sin \frac{n \pi t}{\tau}, \quad 0<t<\tau .
$$

Finally, the half-range Fourier cosine coefficients are

$$
a_{0}=\frac{3}{2}, a_{n}=\frac{2}{\tau} \int_{0}^{\tau}\left(\frac{t}{2 \tau}+\frac{1}{2}\right) \cos \frac{n \pi t}{\tau} d t=\frac{\cos n \pi-1}{n^{2} \pi^{2}},
$$

and so the Fourier cosine series reads

$$
f(t)=\frac{3}{4}+\frac{2}{\pi^{2}} \sum_{n=1,3,5}^{\infty} \frac{1}{n^{2}} \cos \frac{n \pi t}{\tau}, \quad 0 \leqslant t \leqslant \tau .
$$

All three series are shown in Figure 8.7.

\subsection{Complex Form of Fourier Series}

As we noted at the beginning of Section 8.2, Fourier series can also be expressed in a complex form: 


$$
\begin{aligned}
f(x) & =\frac{a_{0}}{2}+\sum_{n=1}^{\infty}\left[a_{n} \cos n x+b_{n} \sin n x\right] \\
& =\frac{a_{0}}{2}+\sum_{n=1}^{\infty}\left[a_{n} \frac{e^{i n x}+e^{-i n x}}{2}+b_{n} \frac{e^{i n x}-e^{-i n x}}{2 i}\right] \\
& =\frac{a_{0}}{2}+\sum_{n=1}^{\infty}\left[\frac{a_{n}-i b_{n}}{2} e^{i n x}+\frac{a_{n}+i b_{n}}{2} e^{-i n x}\right]
\end{aligned}
$$

or,

$$
f(x)=\sum_{n=-\infty}^{\infty} c_{n} e^{i n x}
$$

where

$$
c_{n}=\left\{\begin{array}{cl}
\frac{a_{0}}{2}, & n=0 \\
\frac{a_{n}-i b_{n}}{2}, & n>0 \\
\frac{a_{|n|}+i b_{|n|}}{2}, & n<0 .
\end{array}\right.
$$

From the formulas for $a_{n}$ and $b_{n}$ we find the corresponding Euler formula for $c_{n}$ :

$$
c_{n}=\frac{1}{2 \pi} \int_{-\pi}^{\pi} f(x) e^{-i n x} d x .
$$

Alternatively, this formula can be obtained by multiplying the series (8.5.1) by $e^{-i m x}$, integrating, and using the (orthogonality) relation

$$
\int_{-\pi}^{\pi} e^{i n x} e^{-i m x} d x=\left\{\begin{array}{cc}
0, & n \neq m \\
2 \pi, & n=m .
\end{array}\right.
$$

For an arbitrary period $2 L$ and interval $-L \leqslant x \leqslant L$, (8.5.1) and (8.5.3) become

$$
f(x)=\sum_{n=-\infty}^{\infty} c_{n} e^{i \frac{n \pi x}{L}}
$$

with

$$
c_{n}=\frac{2}{L} \int_{-L}^{L} f(x) e^{-i \frac{n \pi x}{L}} d x .
$$

Example: Fourier series have important applications in connection with differential equations. As an introduction we shall confine ourselves to an example involving an 
ordinary differential equation. Applications in connection with partial differential equations will be considered in a subsequent chapter.

The forced oscillations of a body of mass $m$ on a spring are governed by the equation

$$
m \frac{d^{2} x}{d t^{2}}+r \frac{d x}{d t}+k x=f(t)
$$

where $k$ is the spring constant, $r$ is the damping coefficient, and $f(t)$ is the external force. The general solution to this equation can be written (see Chapter 9) as the general solution to the associated homogeneous equation

$$
m \frac{d^{2} x}{d t^{2}}+r \frac{d x}{d t}+k x=0
$$

plus a particular solution of the non-homogeneous equation. For the homogeneous equation, substitution of the ansatz $x=e^{p t}$ results in the characteristic equation

$$
\left(m p^{2}+r p+k\right) e^{p t}=0
$$

with roots

$$
p=\frac{-r \pm \sqrt{r^{2}-4 m k}}{2 m} .
$$

All real physical systems have positive damping coefficients and so their homogeneous solutions are either damped sinusoidal functions or decaying exponentials:

$$
c_{1} e^{-(r / 2 m) t} \cos \sqrt{k / m-r^{2} / 4 m^{2}} t+c_{2} e^{-(r / 2 m) t} \sin \sqrt{k / e m-r^{2} / 4 m^{2}} t
$$

or,

$$
c_{1} \exp \left[\frac{-r+\sqrt{r^{2}-4 m k}}{2 m} t\right]+c_{2} \exp \left[\frac{-r-\sqrt{r^{2}-4 m k}}{2 m} t\right] .
$$

To determine a particular nonhomogeneous solution, we note that substitution of the ansatz

$$
\chi(t)=X e^{i \omega t}
$$

with an undetermined coefficient $X$ gives

$$
m(i \omega)^{2} X e^{i \omega t}+r(i \omega) X e^{i \omega t}+k X e^{i \omega t}=A e^{i \omega t},
$$

or,

$$
X=\frac{A}{-m \omega^{2}+i r \omega+k}
$$

and hence, leads immediately to the general solution

$$
x(t)=\frac{A}{-m \omega^{2}+i r \omega+k} e^{i \omega t}+c_{1} x_{1}(t)+c_{2} x_{2}(t)
$$

where $x_{1}(t)$ and $x_{2}(t)$ are the homogeneous solutions noted above. The last two terms are called "transients" and approach zero as time increases. Thus, the solution decays 
to a "steady state" sinusoidal response oscillating at the same frequency as the driving term $A e^{i \omega t}$. This oscillation never dies out as long as the driving force is applied; its amplitude $X$ is fixed.

Now let us suppose that the driving force $f(t)$ is not sinusoidal but that it is periodic. The steady state response of the system will again mirror the periodicity of the imposed force and to find an explicit expression for it we simply assume Fourier expansions for $f(t)$ and $x(t)$ :

$$
f(t)=\sum_{n=-\infty}^{\infty} A_{n} e^{i n \omega t} \text { and } x(t)=\sum_{n=-\infty}^{\infty} c_{n} e^{i n \omega t}, \omega=2 \pi / T .
$$

Assuming further that the series for $x(t)$ can be differentiated term by term the necessary number of times, we can substitute these two series plus

$$
\frac{d x}{d t}=\sum_{n=-\infty}^{\infty} i n \omega c_{n} e^{i n \omega t} \text { and } \frac{d^{2} x}{d t^{2}}=\sum_{n=-\infty}^{\infty}\left(-n^{2} \omega^{2}\right) c_{n} e^{i n \omega t},
$$

into the differential equation. Then, invoking orthogonality, we can equate the coefficients with the same exponential $e^{i n \omega t}$ on both sides. The result is

$$
\left(-n^{2} \omega^{2} m+i n \omega r+k\right) c_{n}=A_{n}
$$

or,

$$
c_{n}=\frac{A_{n} / m}{\left(\omega_{0}^{2}-n^{2} \omega^{2}\right)+2 \lambda n \omega i}
$$

where $\omega_{0}^{2}=k / m$ is the natural frequency of the oscillator and $\lambda=r / 2 m$ is the system's damping factor. It only remains to determine the Fourier coefficients for $f(t)$ by applying

$$
A_{n}=\frac{1}{T} \int_{-T / 2}^{T / 2} f(t) e^{-i n \omega t} d t
$$

Thus, we obtain the steady state solution as a superposition of sinusoidal functions with frequencies that are integral multiples of $2 \pi / T$, $T$ being the period of the driving force.

If the frequency of one of these functions is close to the natural frequency $\omega_{0}$ of the system, a resonance effect occurs because of the near cancellation in the denominator of $c_{n}$; that function then becomes the dominant part of the system's response to the imposed force. To offer a concrete illustration of this, let $m=1(\mathrm{~kg}), r=0.02(\mathrm{~kg} / \mathrm{sec})$, and $k=25\left(\mathrm{~kg} / \mathrm{sec}^{2}\right)$, so that the equation of motion becomes

$$
\frac{d^{2} x}{d t^{2}}+0.02 \frac{d x}{d t}+25 x=f(t)
$$

where $f(t)$ is measured in $\mathrm{kg} \cdot \mathrm{m} / \mathrm{sec}^{2}$. Furthermore, let

$$
f(t)=\left\{\begin{array}{cl}
t+\pi / 2 & \text { for }-\pi<t<0, \\
-t+\pi / 2 & \text { for } 0<t<\pi
\end{array}\right.
$$


with $f(t+2 \pi)=f(t)$. Representing $f(t)$ by a Fourier series, we find $A_{n}=\frac{1}{2 \pi}\left[\int_{-\pi}^{0}(t+\pi / 2) e^{-i n t} d t+\int_{0}^{\pi}(-t+\pi / 2) e^{-i n t} d t\right]=\frac{1}{2 \pi} \int_{0}^{\pi}(-t+\pi / 2)\left[e^{-i n t}+e^{i n t}\right] d t$ or,

$$
A_{n}=\frac{1}{\pi} \int_{0}^{\pi}(-t+\pi / 2) \cos n t d t=\frac{1}{n^{2} \pi}\left[1-(-1)^{n}\right]
$$

and thus,

$$
f(t)=\frac{2}{\pi} \sum_{n=-\infty, n \text { odd }}^{\infty} \frac{1}{n^{2}} e^{i n t} .
$$

From the preceding analysis we know that the oscillator's response to this force will be a displacement $x(t)$ with Fourier coefficients

$$
c_{n}=\frac{A_{n} / m}{\left(\omega_{0}^{2}-n^{2}\right)+2 \lambda n i}, \quad n= \pm 1, \pm 3, \pm 5, \ldots
$$

where $\omega_{0}^{2}=k / m=25, \lambda=r / 2 m=0.01$. Thus,

$$
c_{n}=\frac{2}{n^{2} \pi} \frac{\left(25-n^{2}\right)-0.02 n i}{\left(25-n^{2}\right)^{2}+(0.02 n)^{2}}, \quad n= \pm 1, \pm 3, \pm 5, \ldots
$$

which leads directly to

$$
x(t)=\sum_{n=1,3,5, \ldots}^{\infty}\left[a_{n} \cos n t+b_{n} \sin n t\right]
$$

where

$$
a_{n}=\frac{4}{n^{2} \pi} \frac{25-n^{2}}{\left(25-n^{2}\right)^{2}+(0.02 n)^{2}} \text { and } b_{n}=\frac{0.08}{n^{2} \pi} \frac{1}{\left(25-n^{2}\right)^{2}+(0.02 n)^{2}} .
$$

The amplitude of each (complex) mode of oscillation is

$$
\left|c_{n}\right|=\frac{1}{2} \sqrt{a_{n}^{2}+b_{n}^{2}}=\frac{2}{n^{2} \pi} \frac{1}{\sqrt{\left(25-n^{2}\right)^{2}+(0.02 n)^{2}}} .
$$

Some numerical values are

$$
\left|c_{1}\right|=0.0265,\left|c_{3}\right|=0.0044,\left|c_{5}\right|=0.2550,\left|c_{7}\right|=0.0006,\left|c_{9}\right|=0.0001 .
$$

Thus, the cancellation in the denominator of $\left|c_{5}\right|$ results in the $n= \pm 5$ modes dominating and in fact, since $a_{5}=0$, results in the sin $5 t$ term dominating the Fourier series for $x(t)$. This means that the steady state response of the system is almost a pure sine wave with frequency five times that of the driving force. 


\subsection{Transition to Fourier Transforms}

As we noted in the context of analytic continuation, integral representations of functions are often more useful than series representations. Typically, they assume the form of an integral transform (see Section 5.4)

$$
f(z)=\int_{C} K(z, \zeta) g(\zeta) d \zeta
$$

where the function $K(z, \zeta)$ is called the kernel of the representation. We noted further that a commonly used kernel is named after Fourier, has the functional dependence $K(z, \zeta)=\frac{1}{2 \pi} e^{-i z \zeta}$ and corresponds to a choice of contour $C$ that runs along the entire real axis, $-\infty<\zeta<\infty$. The representation that results is

$$
f(z)=\frac{1}{2 \pi} \int_{-\infty}^{\infty} e^{-i z \zeta} g(\zeta) d \zeta .
$$

The similarity in both name and form to the representation afforded by a complex Fourier series suggests that there ought to be a connection and, at the heuristic level at least, there is.

Our starting point is equations (8.5.4) and (8.5.5):

$$
\begin{array}{r}
f(x)=\sum_{n=-\infty}^{\infty} c_{n} e^{i \frac{n \pi x}{L}}, \quad-L \leqslant x \leqslant L, \\
c_{n}=\frac{2}{L} \int_{-L}^{L} f(x) e^{-i \frac{n \pi x}{L}} d x .
\end{array}
$$

If we now define

$$
k_{n} \equiv \frac{n \pi}{L} \quad \text { and } \quad \Delta k \equiv \frac{\pi}{L}=k_{n+1}-k_{n},
$$

these equations can be rewritten as

$$
\begin{aligned}
f(x) & =\sum_{n=-\infty}^{\infty} c_{L}\left(k_{n}\right) e^{i k_{n} x} \Delta k \text { with } \\
c_{L}\left(k_{n}\right) & =\frac{L}{\pi} c_{n}=\frac{1}{2 \pi} \int_{-L}^{L} f(x) e^{-i k_{n} x} d x .
\end{aligned}
$$

Then, taking the limit as $L \rightarrow \infty$, we have

$$
\begin{gathered}
C(k)=\lim _{L \rightarrow \infty} c_{L}(k)=\frac{1}{2 \pi} \int_{-\infty}^{\infty} f(x) e^{-i k x} d x \text { and } \\
f(x)=\int_{-\infty}^{\infty} C(k) e^{i k x} d k
\end{gathered}
$$


which comprise a Fourier transform pair. By modern convention, the Fourier transform pair is defined by replacing $C(k)$ by $F(k)=\sqrt{2 \pi} C(-k)$; thus,

$$
\begin{aligned}
& F(k)=\frac{1}{\sqrt{2 \pi}} \int_{-\infty}^{\infty} f(x) e^{i k x} d x \text { which yields the representation } \\
& f(x)=\frac{1}{\sqrt{2 \pi}} \int_{-\infty}^{\infty} F(k) e^{-i k x} d k
\end{aligned}
$$

The function $F(k)$ is identified as the Fourier transform of $f(x)$ and $f(x)$ as the inverse Fourier transform of $F(k)$; symbolically, we write

$$
F(k)=\mathcal{F}\{f(x)\} \text { and } f(x)=\mathcal{F}^{-1}\{F(k)\} .
$$

By construction, the representation of $f(x)$ that this affords is intended to be valid for all real $x,-\infty<x<\infty$, and periodicity is no longer a consideration.

Not surprisingly, each theorem on the convergence properties of Fourier series has a Fourier transform counterpart. Thus, the pointwise convergence of (8.6.2) is addressed by the Fourier integral theorem.

Theorem: Let $f(x)$ be absolutely integrable on $-\infty<x<\infty$. Then,

$$
\frac{1}{2}[f(x+0)+f(x-0)]=\frac{1}{2 \pi} \int_{-\infty}^{\infty} d k \int_{-\infty}^{\infty} d \xi f(\xi) e^{i k(\xi-x)}
$$

provided that $f(\xi)$ is of bounded variation on an interval $a \leqslant \xi \leqslant b$ that includes the point $\xi=x$. Moreover, if the function is continuous on this interval, the integral on the right hand side of (8.6.3) converges uniformly to $f(x)$ for $a \leqslant x \leqslant b$.

Notice that this theorem does not reflect the striking symmetry between $f(x)$ and its Fourier transform $F(k)$; the properties of $F(k)$ are not even mentioned. While a function and its Fourier coefficients are quite different mathematical objects, a function and its Fourier transform are objects of exactly the same type and so the reciprocity implied by the equations in (8.6.2) is of considerable interest. It is addressed by Plancherel's theorem which, as the Fourier transform counterpart of Parseval's theorem, also addresses sufficient conditions for convergence in the mean.

Theorem: Let $f(x)$ be ( Lebesgue ) square integrable on $-\infty<x<\infty$. Then, the integral

$$
F(k, L)=\frac{1}{\sqrt{2 \pi}} \int_{-L}^{L} f(x) e^{i k x} d x
$$

converges in the mean as $L \rightarrow \infty$ to a function $F(k)$ which is itself square integrable on $-\infty<k<\infty$. Furthermore, the integral

$$
f(x, L)=\frac{1}{\sqrt{2 \pi}} \int_{-L}^{L} F(k) e^{-i k x} d k
$$


converges in the mean to $f(x)$ and

$$
\int_{-\infty}^{\infty}|f(x)|^{2} d x=\int_{-\infty}^{\infty}|F(k)|^{2} d k
$$

\subsection{Examples of Fourier Transforms}

Example 1. Consider the function $f(x)=\frac{a}{a^{2}+x^{2}}$. From equation (8.6.2) its Fourier transform is given by

$$
F(k)=\frac{a}{\sqrt{2 \pi}} \int_{-\infty}^{\infty} \frac{e^{i k x}}{a^{2}+x^{2}} d x .
$$

This can be evaluated by means of the calculus of residues. The function $f(z)=$ $\frac{1}{a^{2}+z^{2}}$ satisfies the conditions specified in the theorem of Section 3.3.3 and so equations (3.3.11) and (3.3.12) apply:

$$
\int_{-\infty}^{\infty} f(x) e^{i k x} d x=\left\{\begin{aligned}
2 \pi i \sum_{+} \operatorname{Res}\left[f(z) e^{i k z}\right], & k>0 \\
-2 \pi i \sum_{-} \operatorname{Res}\left[f(z) e^{i k z}\right], & k<0 .
\end{aligned}\right.
$$

There are simple poles at $z= \pm i a$ and the residues there are

$$
\operatorname{Res}\left[\frac{e^{i k z}}{a^{2}+z^{2}}\right]_{z= \pm i a}=\left.\frac{e^{i k z}}{z \pm i a}\right|_{z= \pm i a}=\frac{e^{\mp k a}}{ \pm 2 i a} .
$$

Thus,

$$
F(k)=\sqrt{\frac{\pi}{2}} \cdot \begin{cases}e^{-k a}, & k>0 \\ e^{k a}, & k<0\end{cases}
$$

or, $F(k)=\sqrt{\frac{\pi}{2}} e^{-|k| a}$.

Verifying that equation (8.6.3)) holds, we note that

$$
\begin{aligned}
\frac{1}{\sqrt{2 \pi}} \int_{-\infty}^{\infty} F(k) e^{-i k x} d k & =\frac{1}{\sqrt{2} \pi} \sqrt{\frac{\pi}{2}}\left[\int_{-\infty}^{0} e^{k a-i k x} d k+\int_{0}^{\infty} e^{-k a-i k x} d k\right] \\
& =\frac{1}{2}\left[\frac{1}{a-i x}+\frac{1}{a+i x}\right]=\frac{a}{a^{2}+x^{2}}=f(x)
\end{aligned}
$$

as required.

Notice that if the parameter $a$ is small, $f(x)$ will be sharply peaked about $x=$ $0 ; F(k)$ on the other hand will be relatively spread out on either side of its maximum which occurs at $k=0$. If $a$ is large, the converse obtains: $f(x)$ is flattened while $F(k)$ is 
sharply peaked. This contrast in behaviour between the members of a Fourier transform pair is characteristic and is the mathematical basis for the Heisenberg uncertainty principle in quantum mechanics. We shall see it recur even more dramatically in each of the next two examples.

Example 2. Suppose that we now take $f(x)$ to be a Gaussian probability function: $f(x)=e^{-a x^{2}}, a=$ constant $>0$. Its Fourier transform is given by

$$
F(k)=\frac{1}{\sqrt{2 \pi}} \int_{-\infty}^{\infty} e^{i k x-a x^{2}} d x
$$

which, apart from the normalization factor $\frac{1}{\sqrt{2 \pi}}$, is just Gauss's Integral which was evaluated in Section 3.3.6. That result gives us

$$
F(k)=\frac{1}{\sqrt{2 a}} e^{-k^{2} / 4 a}
$$

which is also a Gaussian but one with a dependence on $a$ that is the inverse of that enjoyed by $f(x)$.

In quantum mechanics it is the squared modulus of the transform pair that has physical significance; $\left(|f(x)|^{2} d x\right.$ and $|F(k)|^{2} d k$ are the probabilities that a particle or system of particles can be localized with position $x$ and wave number $k$, respectively). The function $|f(x)|^{2}=e^{-2 a x^{2}}$ decreases from its maximum of 1 at $x=0$ to a value of $\frac{1}{e}$ at $x= \pm \frac{1}{\sqrt{2 a}}$. Thus, we take its "width" to be $\Delta x=\sqrt{\frac{2}{a}}$. Similarly, the width of $|F(k)|^{2}=\frac{1}{2 a} e^{-k^{2} / 2 a}$ is $\Delta k=2 \sqrt{2 a}$ and so,

$$
\Delta x \cdot \Delta k=4 \gtrsim O(1)
$$

which, as indicated, is a number of order 1 .

Another common transform pair is composed of the functions

$$
f(x)=\left\{\begin{array}{cl}
\frac{1}{\sqrt{a}}, & -a / 2 \leqslant x \leqslant a / 2 \\
0, & |x|>a / 2 .
\end{array}\right.
$$

and

$$
F(k)=\frac{1}{\sqrt{2 \pi}} \int_{-a / 2}^{a / 2} \frac{1}{\sqrt{a}} e^{i k x} d x=\sqrt{\frac{2}{\pi a}} \frac{\sin a k / 2}{k} .
$$

In this case we can take $\Delta x$ and $\Delta k$ to be the distances between the central zeros of $|f(x)|^{2}$ and $|F(k)|^{2}$, respectively. Since the zeros are the same as the central zeros of $f(x)$ and $F(k)$, we find $\Delta x=a$ and $\Delta k=\frac{4 \pi}{a}$ and so recover the relation $\Delta x \cdot \Delta k \gtrsim O(1)$. Because $\Delta x$ and $\Delta k$ can be identified with an uncertainty in assigning to $x$ and $k$ their most probable values, this has come to be called the (Heisenberg) uncertainty relation. Example 3. As a final example, we shall consider a fairly typical function of time

$$
f(t)=\left\{\begin{array}{cc}
0, & t<0 \\
e^{-t / T} \sin \omega_{0} t, & t \geqslant 0
\end{array} .\right.
$$


Physically, this function might represent the displacement of a damped harmonic oscillator, or the electric field in a radiated electromagnetic wave, or the current in an antenna, just to name three possibilities.

The Fourier transform of $f(t)$ is

$$
F(\omega)=\frac{1}{\sqrt{2 \pi}} \int_{0}^{\infty} e^{-t / T} \sin \omega_{0} t e^{i \omega t} d t=\frac{1}{2 \sqrt{2 \pi}}\left[\frac{1}{\omega+\omega_{0}+i / T}-\frac{1}{\omega-\omega_{0}+i / T}\right]
$$

Once $f(t)$ is identified, we can use Parseval's equation (Plancherel's Theorem) to deduce a complementary physical meaning for $F(\omega)$. For example, if $f(t)$ is a radiated electric field, the radiated power will be proportional to $|f(t)|^{2}$ and the total energy radiated will be proportional to $\int_{-\infty}^{\infty}|f(t)|^{2} d t$. But, according to Parseval's equation , this is equal to $\int_{-\infty}^{\infty}|F(\omega)|^{2} d \omega$. Thus, to within a multiplicative constant, $|F(\omega)|^{2}$ must be the energy radiated per unit frequency interval .

Suppose that $T$ is very large so that $\omega_{0} T \gg 1$. Then, as happened with the resonating harmonic oscillator of Section 8.5 where one frequency or term in the Fourier series dominated over all the others, the "frequency spectrum" defined by $F(\omega)$ is sharply peaked about $\omega= \pm \omega_{0}$. For example, near $\omega=\omega_{0}$,

$$
F(\omega) \approx-\frac{1}{2 \sqrt{2 \pi}} \frac{1}{\omega-\omega_{0}+i / T},
$$

and,

$$
|F(\omega)|^{2} \approx \frac{1}{8 \pi} \frac{1}{\left(\omega-\omega_{0}\right)^{2}+1 / T^{2}} .
$$

When $\omega=\omega_{0} \pm 1 / T$, the radiated energy $|F(\omega)|^{2}$ is down by a factor of $\frac{1}{2}$ from its peak value. Thus, the width of the peak at half-maximum, which is a measure of the uncertainty in the frequency of the radiation, is given by $\Gamma=2 / T$. On the other hand, $T$ is the time for the amplitude of the oscillator or of the radiated wave to "decay" by a factor of $e^{-1}$ and so, is a measure of their mean lifetime which, in turn, is a measure of the uncertainty in the time of oscillation or of emission of the radiation. Thus, we recover another (classical) uncertainty relation:

$$
\Delta t \cdot \Delta \omega=T \cdot \frac{2}{T} \gtrsim O(1)
$$

\subsection{The Dirac Delta Function and Transforms of Distributions}

A reordering of the integrations that appear in the statement of the Fourier Integral Theorem (equation (8.6.3)) gives rise to a very suggestive result. Assuming continuity for $f(x)$, (8.6.3) reads

$$
f(x)=\frac{1}{2 \pi} \int_{-\infty}^{\infty} d k \int_{-\infty}^{\infty} d \xi f(\xi) e^{i k(\xi-x)}
$$


which, after reordering, yields

$$
f(x)=\int_{-\infty}^{\infty} d \xi f(\xi) \delta(\xi-x)
$$

where

$$
\delta(\xi-x)=\frac{1}{2 \pi} \int_{-\infty}^{\infty} d k e^{i k(\xi-x)}
$$

At this juncture we have no idea about the legitimacy of reversing the order of integration and, given that the improper integral in (8.8.3) is not defined in any conventional sense, it does look a bit questionable. Nevertheless, let us ignore such niceties for the time being and focus on the intriguing fact that, if it does exist, $\delta(\xi-x)$ is the continuous variable generalization of the Kronecker delta function

$$
\delta(m, n)= \begin{cases}1, & m=n \\ 0, & m \neq n\end{cases}
$$

That is to say, just as $\delta(m, n)$ picks out the $n^{\text {th }}$ term from a summation over $m$,

$$
f_{n}=\sum_{m=-\infty}^{\infty} f_{m} \delta(m, n),
$$

$\delta(\xi-x)$ selects and delivers the value at $\xi=x$ of the function that multiplies it in a summation over the continuous variable $\xi$.

A function with this property is called a Dirac delta function and was introduced by Paul Dirac in his landmark formulation of quantum mechanics that unified the earlier Heisenberg and Schrödinger pictures of quantum phenomena.

It is clear from (8.8.3) that the delta function depends only on the difference $\xi-$ $x$ and not on $\xi$ and $x$ individually. Moreover, equation (8.8.2) tells us that the delta function is normalized for, setting $f(x) \equiv 1$, we have

$$
1=\int_{-\infty}^{\infty} \delta(\xi-x) d \xi
$$

This immediately raises the question of what $\delta(\xi-x)$ "looks like" when it is plotted. From equation (8.8.2) we see that the function $f(\xi)$ can be modified anywhere except at the point $\xi=x$ without affecting the result of the integration. This implies that $\delta(\xi-x)$ must be zero everywhere except in an infinitesimal neighbourhood of $\xi=x$. Equation (8.8.4) then suggests that

$$
1=\lim _{\varepsilon \rightarrow 0} \int_{x-\varepsilon}^{x+\varepsilon} \delta(\xi-x) d \xi
$$


and hence, that $\delta(\xi-x) \rightarrow \infty$ as $\xi \rightarrow x$. In other words, we can think of the delta function as having the mathematically ill-defined properties

$$
\delta(\xi-x)=\left\{\begin{array}{cl}
0 & \text { for all } \xi \neq x \\
\infty & \text { for } \xi=x .
\end{array}\right.
$$

Evidently, neither this "equality" nor equation (8.8.2) from which it was deduced can be used as a formal definition of the Dirac delta function. Nevertheless, they do offer an intuitive appreciation of what a delta function is as well as some idea of how to make use of them.

Another aid for our intuition and one that takes us closer to a formal definition of the Dirac delta function is to think of it as the limit of a sequence of functions that are strongly peaked about $\xi=x$ and satisfy the condition

$$
\lim _{n \rightarrow \infty} \int_{-\infty}^{\infty} \delta_{n}(\xi-x) f(\xi) d \xi=f(x)
$$

for all suitably behaved functions $f(x)$. Such sequences are called delta sequences.

For notational convenience, we are now going to reverse the roles of the symbols $\xi$ and $x$ and set $\xi=0$. Equation (8.8.6) then becomes

$$
\lim _{n \rightarrow \infty} \int_{-\infty}^{\infty} \delta_{n}(x) f(x) d x=f(0)
$$

where $\left\{\delta_{n}(x)\right\}$ is a sequence of functions that are sharply peaked about $x=0$.

Some examples of delta sequences are:

1. $\delta_{n}(x)=\left\{\begin{array}{cl}0 & \text { for }|x| \geqslant 1 / n \\ n / 2 & \text { for }|x|<1 / n\end{array}\right.$

2. $\delta_{n}(x)=\frac{n}{\sqrt{\pi}} e^{-n^{2} x^{2}}$;

3. $\delta_{n}(x)=\frac{n}{\pi} \frac{1}{1+n^{2} x^{2}}$;

4. $\delta_{n}(x)=\frac{\sin n x}{\pi x}=\frac{1}{2 \pi} \int_{-n}^{n} e^{i k x} d k$;

5. $\delta_{n}(x)=\frac{1}{n \pi} \frac{\sin ^{2} n x}{x^{2}}$.

By definition these all satisfy condition (8.8.7). We shall verify this for the first and third sequences. Notice that the fourth sequence links us back to the Fourier transform (8.8.3) that initiated our interest in the delta function.

Using the mean value theorem, substitution of sequence \#1 into the integral on the left hand side of (8.8.7) yields

$$
\int_{-\infty}^{\infty} \delta_{n}(x) f(x) d x=\int_{-1 / n}^{1 / n} \frac{n}{2} f(x) d x=\frac{2}{n} \cdot \frac{n}{2} f\left(x_{m}\right)
$$




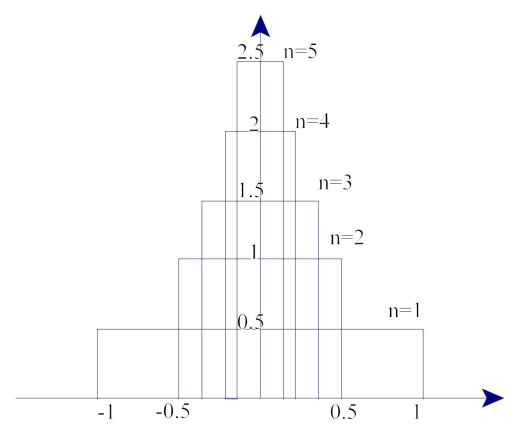

Figure 8.8: $\delta$-sequence \#1

where $-1 / n \leqslant x_{m} \leqslant 1 / n$. Clearly, as $n \rightarrow \infty, x_{m} \rightarrow 0$ and so,

$$
\lim _{n \rightarrow \infty} \int_{-\infty}^{\infty} \delta_{n}(x) f(x) d x=f(0)
$$

as required.

In the case of sequence \#3, the relevant integral is

$$
\int_{-\infty}^{\infty} \frac{n}{\pi} \frac{1}{1+n^{2} x^{2}} f(x) d x
$$

which admits evaluation by residue calculus. Provided that the continuation of the test function, $f(z)$, is meromorphic in the upper half-plane and that $|f(z)|$ is bounded as $|z| \rightarrow \infty$, we can use equation (3.3.4) and write

$$
\int_{-\infty}^{\infty} \frac{n}{\pi} \frac{1}{1+n^{2} x^{2}} f(x) d x=2 \pi i \sum_{+} \operatorname{Res}\left[\delta_{n}(z) f(z)\right]
$$

where $\sum_{+}$denotes the sum over all the poles of $\delta_{n}(z)$ and $f(z)$ in the upper half-plane. However, since $\lim _{n \rightarrow \infty} \delta_{n}(z)=0$ if $z \neq 0$, the contributions to the sum from poles of $f(z)$ will vanish in the limit. Thus,

$$
\lim _{n \rightarrow \infty} \int_{-\infty}^{\infty} \frac{n}{\pi} \frac{1}{1+n^{2} x^{2}} f(x) d x=\lim _{n \rightarrow \infty} 2 \pi i \operatorname{Res}\left[\frac{n}{\pi} \frac{1}{1+n^{2} z^{2}} f(z)\right]_{z=\frac{i}{n}}
$$

or,

$$
\lim _{n \rightarrow \infty} \int_{-\infty}^{\infty} \frac{n}{\pi} \frac{1}{1+n^{2} x^{2}} f(x) d x=\lim _{n \rightarrow \infty} 2 \pi i f\left(\frac{i}{n}\right) \frac{n}{\pi} \frac{1}{2 n^{2} \frac{i}{n}}=\lim _{n \rightarrow \infty} f\left(\frac{i}{n}\right)=f(0)
$$


as required.

The latter sequence often appears in physical applications with $n$ replaced by $\frac{1}{\varepsilon}$ so that $\delta_{n}(x)$ assumes the form

$$
\delta_{\varepsilon}(x)=\frac{\varepsilon}{\pi} \frac{1}{x^{2}+\varepsilon^{2}}=\frac{1}{2 \pi i}\left[\frac{1}{x-i \varepsilon}-\frac{1}{x+i \varepsilon}\right]
$$

and the appropriate limit is now that as $\varepsilon \rightarrow 0$.

While it is helpful to think of the Dirac delta function, $\delta(x)$, as being the limit of any of these sequences, we cannot define it this way because the limits themselves are undefined at $x=0$. Nevertheless, the delta sequences do play a role in defining the delta function not as a conventional function but as a member of a new class of mathematical entities called generalized functions or distributions. These are all defined in terms of sequences of conventional functions by means of a unique limiting process that involves the integrals of these functions "against" an appropriately wellbehaved test function. In the case of $\delta(x)$ this yields a definition that recognizes that the fundamental property of the function is expressed by

$$
\int_{-\infty}^{\infty} \delta(x) f(x) d x=f(0)
$$

Definition: Any sequence of continuous functions $g_{n}(x)$ defines a distribution $g(x)$ if, for any function $f(x)$ which is differentiable everywhere any number of times and which is non-zero only on a bounded set, the limit

$$
\lim _{n \rightarrow \infty} \int_{-\infty}^{\infty} g_{n}(x) f(x) d x \equiv \int_{-\infty}^{\infty} g(x) f(x) d x
$$

exists.

The right hand side of this equation is not a Riemann integral but rather, it denotes the limit of a sequence of Riemann integrals.

Two distributions $g(x)$ and $h(x)$ are equal if the corresponding sequences satisfy

$$
\lim _{n \rightarrow \infty} \int_{-\infty}^{\infty} g_{n}(x) f(x) d x=\lim _{n \rightarrow \infty} \int_{-\infty}^{\infty} h_{n}(x) f(x) d x
$$

for any "test function" $f(x)$ that has the properties specified in the definition. Thus, for example, the delta-sequences $\left\{\delta_{n}(x)\right\}$ all define the same distribution $\delta(x)$ :

$$
\lim _{n \rightarrow \infty} \int_{-\infty}^{\infty} \delta_{n}(x) f(x) d x=\int_{-\infty}^{\infty} \delta(x) f(x) d x=f(0) .
$$

The principal result of the theory of distributions, from the perspective of a physicist, is that they admit manipulation according to the same rules of calculus as apply to 
conventional functions. However, one must remember that they have significance or meaning only as a multiplicative factor in an integrand.

Because of its peculiar properties, the Dirac delta function is an extremely useful artifact of mathematical physics. In addition to arising in a very natural way from a consideration of the representation of functions by means of Fourier series and transforms, they offer a functional expression of the most common idealizations of physics: that particles can be localized at points and rigid bodies can be so rigid that their elastic collisions are mediated by instantaneous impulses. The charge density for a collection of point charges and the force exerted by one perfectly rigid billiard ball on another are necessarily delta functions of position and time, respectively. This, in turn, leads to a central role for the delta function in the Green's Function approach to solving non- homogeneous boundary value problems as we shall see in Chapter 12. Thus, for all these reasons, we shall digress with an exploration of what can be learned about delta functions from an application of the $\delta$-calculus.

The $\delta$-calculus involves the treatment of $\delta(x)$ and its derivatives as though they were conventional functions albeit ones with the "unusual" properties

1. $\int_{-\infty}^{\infty} \delta(x) f(x) d x=f(0)$, and

2. $\delta(x)=\left\{\begin{array}{cc}\infty, & x=0 \\ 0, & x \neq 0\end{array}\right.$ with $\int_{-\infty}^{\infty} \delta(x) d x=1$

It is a shortcut method for obtaining identities that are all derivable by a rigorous but much more onerous approach involving $\delta$-sequences and the limiting processes that define distributions.

To begin with we shall "prove" that

$$
\delta(x)=\frac{d}{d x} \theta(x)
$$

where $\theta$ is the step function

$$
\theta(x)= \begin{cases}1, & x>0 \\ 0, & x<0\end{cases}
$$

Using a continuous but otherwise arbitrary test function $f(x)$, we compose the integral

$$
\int_{-\infty}^{\infty} \frac{d \theta(x)}{d x} f(x) d x
$$

and proceed with integration by parts. We find immediately that

$$
\int_{-\infty}^{\infty} \frac{d \theta(x)}{d x} f(x) d x=\left.\theta(x) f(x)\right|_{-\infty} ^{\infty}-\int_{-\infty}^{\infty} \theta(x) f^{\prime}(x) d x=f(\infty)-\int_{0}^{\infty} f^{\prime}(x) d x=f(0)=\int_{-\infty}^{\infty} \delta(x) f(x) d x
$$

which establishes the identity (8.8.11). 
Similarly, by integrating

$$
\int_{-\infty}^{\infty} \delta^{\prime}(x) f(x) d x
$$

by parts, we find

$$
\int_{-\infty}^{\infty} \delta^{\prime}(x) f(x) d x=\left.\delta(x) f(x)\right|_{-\infty} ^{\infty}-\int_{-\infty}^{\infty} \delta(x) f^{\prime}(x) d x
$$

and hence,

$$
\int_{-\infty}^{\infty} \delta^{\prime}(x) f(x) d x=-f^{\prime}(0)
$$

More generally,

$$
\int_{-\infty}^{\infty} \frac{d^{m} \delta(x)}{d x^{m}} f(x) d x=(-1)^{m} \frac{d^{m} f(0)}{d x^{m}}
$$

Next, we note that

$$
\int_{-\infty}^{\infty} x \delta(x) f(x) d x=0 \text { and } \int_{-\infty}^{\infty} x \delta^{\prime}(x) f(x) d x=f(0)=\int_{-\infty}^{\infty} \delta(x) f(x) d x
$$

for arbitrary continuous functions $f(x)$, and deduce the identities

$$
\begin{gathered}
x \delta(x)=0 \\
x \delta^{\prime}(x)=\delta(x)
\end{gathered}
$$

A further identity is

$$
\delta(a x)=\frac{1}{|a|} \delta(x), \quad a \neq 0 .
$$

To establish it, we use the usual continuous test function $f(x)$ and evaluate

$$
\int_{-\infty}^{\infty} \delta(a x) f(x) d x=\left\{\begin{array}{cc}
\int_{-\infty}^{\infty} \delta(\xi) f(\xi / a) \frac{1}{a} d \xi=\frac{1}{a} f(0), & a>0 \\
\int_{-\infty}^{\infty} \delta(\xi) f(\xi / a) \frac{1}{a} d \xi=-\frac{1}{a} f(0), & a<0
\end{array} \quad=\int_{-\infty}^{\infty} \frac{1}{|a|} \delta(x) f(x) d x .\right.
$$

Evidently, $\delta(x)$ is an even function since, from (8.8.16), we have

$$
\delta(-x)=\delta(x)
$$


This comes as no great surprise given that the $\delta$-sequences all consist of even functions.

A useful extension of (8.8.16) is the identity

$$
\delta\left(x^{2}-a^{2}\right)=\frac{1}{2 a}[\delta(x-a)+\delta(x+a)], \quad a>0 .
$$

Whereas the argument of the $\delta$-function on the left hand side of (8.8.16) is a linear function of $x$, we now have a quadratic function as an argument. The derivation has the usual starting point: we compose the integral $\int_{-\infty}^{\infty} \delta\left(x^{2}-a^{2}\right) f(x) d x$ for arbitrary continuous $f(x)$. However, we now invoke the property that $\delta(\xi)=0$ except at $\xi=0$ to write

$$
\int_{-\infty}^{\infty} \delta\left(x^{2}-a^{2}\right) f(x) d x=\int_{-a-\varepsilon}^{-a+\varepsilon} \delta\left(x^{2}-a^{2}\right) f(x) d x+\int_{a-\varepsilon}^{a+\varepsilon} \delta\left(x^{2}-a^{2}\right) f(x) d x
$$

where $0<\varepsilon<2 a$. Introducing the new variable of integration $\xi=x^{2}-a^{2}$, we have

$$
d x= \begin{cases}d \xi / 2 \sqrt{\xi+a^{2}}, & x>0 \\ -d \xi / 2 \sqrt{\xi+a^{2}} & , x<0\end{cases}
$$

and so,

$$
\begin{aligned}
\delta\left(x^{2}-a^{2}\right) f(x) d x & =\int_{2 \varepsilon a}^{-2 \varepsilon a}-\delta(\xi) f\left(-\sqrt{\xi+a^{2}}\right) \frac{d \xi}{2 \sqrt{\xi+a^{2}}}+\int_{-2 \varepsilon a}^{2 \varepsilon a} \delta(\xi) f\left(\sqrt{\xi+a^{2}}\right) \frac{d \xi}{2 \sqrt{\xi+a}} \\
& =\frac{1}{2 a}[f(-a)+f(a)] \\
& =\frac{1}{2 a} \int_{-\infty}^{\infty}[\delta(x+a)+\delta(x-a)] f(x) d x
\end{aligned}
$$

as required.

This last identity can be extended to cover all cases of a $\delta$-function whose argument is a differentiable function with simple zeros. If $g(x)$ is differentiable everywhere and $g\left(x_{n}\right)=0$ with $g^{\prime}\left(x_{n}\right) \neq 0$ for a countable set of points $x_{n}$ then,

$$
\delta[g(x)]=\sum_{n} \frac{1}{\left|g^{\prime}\left(x_{n}\right)\right|} \delta\left(x-x_{n}\right) .
$$

The $\delta$-calculus can also be used to determine series and integral representations for delta functions. For example, the Fourier coefficients of $\delta(x)$ are $b_{n}=0(\delta(x)$ is an even function) and

$$
a_{n}=\frac{1}{L} \int_{-L}^{L} \delta(x) \cos \frac{n \pi x}{L} d x=\frac{1}{L}
$$


Thus, the Fourier series representation of $\delta(x)$ is

$$
\delta(x)=\frac{1}{2 L}+\frac{1}{L} \sum_{n=1}^{\infty} \cos \frac{n \pi x}{L} .
$$

This is a divergent series, as one might expect, but it possesses the key property

$$
\int_{-L}^{L} \delta(x) f(x) d x=f(0)
$$

as can be seen by multiplying the right hand side of (8.8.20) by an arbitrary continuous function $f(x)$ and integrating term by term from $-L$ to $L$ :

$$
\frac{1}{2 L} \int_{-L}^{L} f(x) d x+\sum_{n=1}^{\infty} \frac{1}{L} \int_{-L}^{L} f(x) \cos \frac{n \pi x}{L} d x=\frac{a_{0}}{2}+\sum_{n=1}^{\infty} a_{n},
$$

where $a_{n}, n=0,1,2, \ldots$ are Fourier coefficients of $f(x)$. If $f(x)$ has the Fourier series

$$
f(x)=\frac{a_{0}}{2}+\sum_{n=1}^{\infty}\left(a_{n} \cos \frac{n \pi x}{L}+b_{n} \sin \frac{n \pi x}{L}\right),
$$

then

$$
\frac{a_{0}}{2}+\sum_{n=1}^{\infty} a_{n}=f(0)
$$

Following exactly the same steps we can determine various other series for the delta function. For instance, if $0<\xi<L$, the Fourier sine and cosine series for $\delta(x-\xi)$ are

$$
\delta(x-\xi)=\frac{2}{L} \sum_{n=1}^{\infty} \sin \frac{n \pi \xi}{L} \sin \frac{n \pi x}{L}
$$

and

$$
\delta(x-\xi)=\frac{1}{L}+\frac{2}{L} \sum_{n=1}^{\infty} \cos \frac{n \pi \xi}{L} \cos \frac{n \pi x}{L},
$$

respectively.

These are called the closure relations for the orthonormal (orthogonal and normalized) sets $\left\{\sqrt{\frac{2}{L}} \sin \frac{n \pi x}{L}\right\}$ and $\left\{\sqrt{\frac{2}{L}} \cos \frac{n \pi x}{L}\right\}$ on the interval $0 \leqslant x \leqslant L$. The integral representation

$$
\delta(x-\xi)=\frac{1}{2 \pi} \int_{-\infty}^{\infty} e^{i k(x-\xi)} d k
$$


that was our initial introduction to the delta function is also a closure relation. In this case, the set of orthonormal functions is $\left\{\sqrt{\frac{1}{2 \pi}} e^{i k x}\right\}$ and their orthogonality and normalization over the interval $-\infty<x<\infty$ is expressed by

$$
\frac{1}{2 \pi} \int_{-\infty}^{\infty} e^{i(k-j) x} d x=\delta(k-j)
$$

where, because $k$ and $j$ are continuous indices, the usual Kronecker delta function has been replaced by a Dirac delta function.

Equation (8.8.23) (and (8.8.24)) can also be interpreted as stating that $\delta(x-\xi)$ and $\sqrt{\frac{1}{2 \pi}} e^{-i k \xi}$ (and $\delta(k-j)$ and $\sqrt{\frac{1}{2 \pi}} e^{-i j x}$ ) comprise Fourier transform pairs. The trigonometrical exponential does not meet the integrability condition specified in either Fourier's Integral Theorem or Plancherel's Theorem. Fortunately, however, this is another apparent impediment that has been removed by appeal to distribution theory. It has been proven that every distribution has a Fourier transform which is itself a distribution. Thus, not only the trigonometrical functions but even polynomials can have well-defined transforms through the expedient of treating them as distributions or generalized functions.

We shall conclude this Section by noting that the concept of a delta function and the $\delta$-calculus can be extended to two or more dimensions via a corresponding extension of the concept of a distribution. Using an arbitrary continuous function of position $f(\vec{r})$ we have the defining equation

$$
f\left(\vec{r}_{0}\right)=\int_{\text {all space }} f(\vec{r}) \delta\left(\vec{r}-\vec{r}_{0}\right) d^{n} r .
$$

In terms of three dimensional Cartesian, spherical polar and cylindrical polar coordinates this becomes

$$
\begin{aligned}
f\left(\vec{r}_{0}\right) & =\int_{-\infty}^{\infty} \int_{-\infty}^{\infty} \int_{-\infty}^{\infty} f(x, y, z) \delta\left(\vec{r}-\vec{r}_{0}\right) d x d y d z \\
& =\int_{0}^{\infty} \int_{-1}^{1} \int_{0}^{2 \pi} f(r, \cos \theta, \varphi) \delta\left(\vec{r}-\vec{r}_{0}\right) r^{2} d r d(\cos \theta) d \varphi \\
& =\int_{0}^{\infty} \int_{0}^{2 \pi} \int_{-\infty}^{\infty} f(\rho, \varphi, z) \delta\left(\vec{r}-\vec{r}_{0}\right) \rho d \rho d \varphi d z
\end{aligned}
$$

from which we deduce 


$$
\begin{aligned}
\delta\left(\vec{r}-\vec{r}_{0}\right) & =\delta\left(x-x_{0}\right) \delta\left(y-y_{0}\right) \delta\left(z-z_{0}\right)=\frac{1}{r^{2}} \delta\left(r-r_{0}\right) \delta\left(\cos \theta-\cos \theta_{0}\right) \delta\left(\varphi-\varphi_{0}\right) \\
& =\frac{1}{\rho} \delta\left(\rho-\rho_{0}\right) \delta\left(\varphi-\varphi_{0}\right) \delta\left(z-z_{0}\right) .
\end{aligned}
$$

An interesting representation of $\delta\left(\vec{r}-\vec{r}_{0}\right)$ and one that should evoke memories of Coulomb's Law is

$$
\delta\left(\vec{r}-\vec{r}_{0}\right)=-\frac{1}{4 \pi} \nabla^{2}\left(\frac{1}{r}\right) .
$$

To derive this relationship we shall locate the origin of our coordinate system at $\vec{r}=\vec{r}_{0}$ and attempt to show that

$$
\int_{\text {all space }} f(\vec{r}) \nabla^{2}\left(\frac{1}{r}\right) d^{3} r=-4 \pi f(0)
$$

for an arbitrary but continuous function $f(\vec{r})$. The integral over all space on the left hand side of this equation can be set equal to the integral over a sphere of volume $\mathrm{V}$ and surface $S$ in the limit as the radius of the sphere $R \rightarrow \infty$. Before taking that limit, however, let us apply the divergence theorem to the vector function $f(\vec{r}) \vec{\nabla}\left(\frac{1}{r}\right)$ :

$$
\int_{V} \vec{\nabla} \cdot\left[f(\vec{r}) \vec{\nabla}\left(\frac{1}{r}\right)\right] d^{3} r=\int_{S}\left[f(\vec{r}) \vec{\nabla}\left(\frac{1}{r}\right)\right] \cdot d \vec{S} .
$$

Thus, since

$$
\vec{\nabla} \cdot\left[f(\vec{r}) \vec{\nabla}\left(\frac{1}{r}\right)\right]=\vec{\nabla} f(\vec{r}) \cdot \vec{\nabla}\left(\frac{1}{r}\right)+f(\vec{r}) \nabla^{2}\left(\frac{1}{r}\right),
$$

we have

$$
\left.\int_{V} f(\vec{r}) \nabla^{2}\left(\frac{1}{r}\right) d^{3} r=\int_{S}\left[f(\vec{r}) \vec{\nabla}\left(\frac{1}{r}\right)\right)\right] \cdot d \vec{S}-\int_{V} \vec{\nabla} f(\vec{r}) \cdot \vec{\nabla}\left(\frac{1}{r}\right) d^{3} r .
$$

But, $\vec{\nabla}\left(\frac{1}{r}\right)=-\frac{1}{r^{2}} \frac{\vec{r}}{r}$ and $\frac{\vec{r}}{r} \cdot \vec{\nabla} f(\vec{r})=\frac{\partial}{\partial r} f(\vec{r})$. Therefore,

$$
\begin{aligned}
\int_{V} f(\vec{r}) \nabla^{2}\left(\frac{1}{r}\right) d^{3} r & =\left.\int_{-1}^{1} \int_{0}^{2 \pi} f(\vec{r})\right|_{r=R}\left(-\frac{1}{r^{2}}\right) r^{2} d \cos \theta d \varphi \\
& -\int_{0}^{R} \int_{-1}^{1} \int_{0}^{2 \pi}\left[-\frac{\partial}{\partial r} f(\vec{r})\right] d r d \cos \theta d \varphi
\end{aligned}
$$

or,

$$
\int_{V} f(\vec{r}) \nabla^{2}\left(\frac{1}{r}\right) d^{3} r=-\left.\int_{-1}^{1} \int_{0}^{2 \pi} f(\vec{r})\right|_{r=R} d \cos \theta d \varphi+\left.\int_{-1}^{1} \int_{0}^{2 \pi} f(\vec{r})\right|_{r=0} ^{r=R} d \cos \theta d \varphi=-4 \pi f(0),
$$

as required.

We shall now return to the subject of Fourier transforms. 


\subsection{Properties of Fourier Transforms}

From the defining integral,

$$
\mathcal{F}\{f(x)\} \equiv F(k)=\frac{1}{\sqrt{2 \pi}} \int_{-\infty}^{\infty} f(x) e^{i k x} d x,
$$

it follows that for real functions $f(x)$

$$
F(-k)=F^{\star}(k) .
$$

This is called the conjugation property and it has two immediate corollaries:

1. $F(k)$ is real if $f(x)$ is an even function,

2. $F(k)$ is imaginary if $f(x)$ is an odd function of $x$.

Multiplying $f(x)$ by $e^{-a x}$ and then calculating the transform, we have

$$
\mathcal{F}\left\{e^{-a x} f(x)\right\}=\frac{1}{\sqrt{2 \pi}} \int_{-\infty}^{\infty} f(x) e^{i(k+i a) x} d x=F(k+i a)
$$

which is called the attenuation property.

Similarly, a displacement of the argument of $f(x)$ results in multiplication of $F(k)$ by a phase (rather than an attenuation) factor:

$$
\mathcal{F}\{f(x-a)\}=\frac{1}{\sqrt{2 \pi}} \int_{-\infty}^{\infty} f(\xi) e^{i k(\xi+a)} d \xi=e^{i k a} F(k)=e^{i k a} \mathcal{F}\{f(x)\} .
$$

Next, let us assume that the transform of the derivative of $f(x), \mathcal{F}\left\{f^{\prime}(x)\right\}$, exists. Then, integrating by parts, we have

$$
\frac{1}{\sqrt{2 \pi}} \int_{-\infty}^{\infty} f^{\prime}(x) e^{i k x} d x=\left.\frac{1}{\sqrt{2 \pi}} f(x) e^{i k x}\right|_{-\infty} ^{\infty}-\frac{i k}{\sqrt{2 \pi}} \int_{-\infty}^{\infty} f(x) e^{i k x} d x .
$$

The existence of its Fourier transform implies either that $f(x) \rightarrow 0$ as $x \rightarrow \pm \infty$ or that $f(x)$ is a distribution. In both cases we lose the integrated term and obtain

$$
\mathcal{F}\left\{f^{\prime}(x)\right\}=-i k \mathcal{F}\{f(x)\} .
$$

This is called the differentiation property and it extends in an obvious way to higher derivatives:

$$
\mathcal{F}\left\{f^{\prime \prime}(x)\right\}=-k^{2} \mathcal{F}\{f(x)\} \ldots \mathcal{F}\left\{f^{(n)}(x)\right\}=(-i)^{n} \mathcal{F}\{f(x)\} .
$$

As we shall see, this has immediate application in the solution of differential equations. 
The converse of the differentiation property arises when we transform the product of $f(x)$ and a power of $x$ :

$$
\mathcal{F}\{x f(x)\}=\frac{1}{2 \pi} \int_{-\infty}^{\infty} f(x) x e^{i k x} d x=\frac{1}{2 \pi} \int_{-\infty}^{\infty} f(x)\left(-i \frac{d}{d k}\right) e^{i k x} d x=-i \frac{d}{d k} \mathcal{F}\{f(x)\},
$$

provided that we can interchange the order of integration and differentiation. This too can be extended to read

$$
\mathcal{F}\left\{x^{n} f(x)\right\}=(-i)^{n} \frac{d^{n}}{d x^{n}} \mathcal{F}\{f(x)\} .
$$

If $F(k)=\mathcal{F}\{f(x)\}$ and $G(k)=\mathcal{F}\{g(x)\}$, then the product $H(k)=F(k) G(k)$ is the Fourier transform of the function

$$
h(x)=\frac{1}{2 \pi} \int_{-\infty}^{\infty} f(\xi) g(x-\xi) d \xi .
$$

The integral in (8.9.9) is of a type that yields a convolution of the two functions in the integrand and so this property is called the convolution theorem. Its proof is straightforward:

$$
\mathcal{F}^{-1}\{F(k) G(k)\}=\frac{1}{\sqrt{2 \pi}} \int_{-\infty}^{\infty} F(k) G(k) e^{-i k x} d k=\frac{1}{2 \pi} \int_{-\infty}^{\infty} F(k) \int_{-\infty}^{\infty} g(\xi) e^{i k \xi} d \xi e^{-i k x} d k
$$

assuming that we can interchange the order of integrations, this becomes

$$
\mathcal{F}^{-1}\{F(k) G(k)\}=\frac{1}{2 \pi} \int_{-\infty}^{\infty} g(\xi) \int_{-\infty}^{\infty} F(k) e^{-i k(x-\xi)} d k d \xi=\frac{1}{\sqrt{2 \pi}} \int_{-\infty}^{\infty} g(\xi) f(x-\xi) d \xi,
$$

as stated .

\subsection{Fourier Sine and Cosine Transforms}

Suppose that we are given an even function of $x, f(x)=f(-x)$. Its Fourier transform then reduces to

$$
\mathcal{F}\{f(x)\} \equiv F(k)=\frac{1}{\sqrt{2 \pi}} \int_{-\infty}^{\infty} f(x) e^{i k x} d x=\sqrt{\frac{2}{\pi}} \int_{0}^{\infty} f(x) \cos k x d x .
$$

Evidently, $F(k)$ is, in turn, an even function of $k$ and so we also have

$$
f(x)=\mathcal{F}^{-1}\{F(k)\}=\sqrt{\frac{2}{\pi}} \int_{0}^{\infty} F(k) \cos k x d k .
$$


Similarly, if $f(x)$ is odd, $f(-x)=-f(x)$, then

$$
F(k)=\sqrt{\frac{2}{\pi}} i \int_{0}^{\infty} f(x) \sin k x d x
$$

and,

$$
f(x)=\mathcal{F}^{-1}\{F(k)\}=\sqrt{\frac{2}{\pi}}(-i) \int_{0}^{\infty} f(x) \sin k x d k .
$$

This suggests that just as we had Fourier cosine and sine series for functions defined on the (half-) interval $0 \leqslant x \leqslant \pi$, we can introduce Fourier cosine and sine transforms for functions defined only on the (half-) interval $0 \leqslant x<\infty$ :

$$
\mathcal{F}_{c}\{f(x)\} \equiv F_{c}(k)=\sqrt{\frac{2}{\pi}} \int_{0}^{\infty} f(x) \cos k x d x
$$

with

$$
\mathcal{F}_{c}^{-1}\left\{F_{c}(k)\right\} \equiv \sqrt{\frac{2}{\pi}} \int_{0}^{\infty} F_{c}(k) \cos k x d k=f(x)
$$

and,

$$
\mathcal{F}_{S}\{f(x)\} \equiv F_{s}(k)=\sqrt{\frac{2}{\pi}} \int_{0}^{\infty} f(x) \sin k x d x
$$

with

$$
\mathcal{F}_{s}^{-1}\left\{F_{s}(k)\right\} \equiv \sqrt{\frac{2}{\pi}} \int_{0}^{\infty} F_{s}(k) \sin k x d x=f(x) .
$$

Note that

$$
\mathcal{F}_{c}\{f(x)\}=\mathcal{F}\left\{f^{(+)}(x)\right\}
$$

and

$$
\mathcal{F}_{s}\{f(x)\}=-i \mathcal{F}\left\{f^{(-)}(x)\right\}
$$

where

$$
f^{(+)}(x)=\left\{\begin{array}{rr}
f(x), & x>0 \\
f(-x), & x<0
\end{array}\right.
$$

is the symmetric extension of $f(x)$ and

$$
f^{(-)}(x)=\left\{\begin{array}{cc}
f(x), & x>0 \\
-f(-x), & x<0
\end{array}\right.
$$


is the antisymmetric extension of $f(x)$. As a result, Fourier sine and cosine transforms possess properties very similar to those of Fourier transforms. Some differences warrant our attention however. For example,

$$
\mathcal{F}_{c}\left\{f^{\prime}(x)\right\}=\sqrt{\frac{2}{\pi}} \int_{0}^{\infty} f^{\prime}(x) \cos k x d x=\left.\sqrt{\frac{2}{\pi}} f(x) \cos k x\right|_{0} ^{\infty}+\sqrt{\frac{2}{\pi}} k \int_{0}^{\infty} f(x) \sin k x d x .
$$

As before, we can assume that $f(x) \rightarrow 0$ as $x \rightarrow \infty$ (or we can treat it as a distribution) and so obtain

$$
\mathcal{F}_{c}\left\{f^{\prime}(x)\right\}=-\sqrt{\frac{2}{\pi}} f(0)+k \mathcal{F}_{s}\{f(x)\}
$$

Similarly, integrating by parts twice and assuming that both $f^{\prime}(x) \rightarrow 0$ and $f(x) \rightarrow 0$ as $x \rightarrow \infty$, we find

$$
\mathcal{F}_{c}\left\{f^{\prime \prime}(x)\right\}=-\sqrt{\frac{2}{\pi}} f^{\prime}(0)-k^{2} \mathcal{F}_{c}\{f(x)\} .
$$

The corresponding relationships for Fourier sine transforms are

$$
\mathcal{F}_{s}\left\{f^{\prime}(x)\right\}=-k \mathcal{F}_{c}\{f(x)\}
$$

and

$$
\mathcal{F}_{s}\left\{f^{\prime \prime}(x)\right\}=\sqrt{\frac{2}{\pi}} k f(0)-k^{2} \mathcal{F}_{s}\{f(x)\} .
$$

It should be noted that transforming derivatives of even order yields a transform of the undifferentiated function and it is a transform of the same type. On the other hand, transforms of derivatives of odd order result in a transform of the other type. This has immediate consequences for the application of Fourier sine and cosine transforms in the solution of differential equations: the equations must contain derivatives of only even or only odd order to avoid mixing the two types of transform. Another way in which these differentiation properties influence the application to differential equations is the "boundary condition" information they require: a knowledge of $f(0)$ in the case of sine transforms and of $f^{\prime}(0)$ for cosine transforms.

The interrelation between Fourier sine and cosine transforms surfaces again in their convolution theorems. If $F_{c}(k)=\mathcal{F}_{c}\{f(x)\}$ and $G_{c}(k)=\mathcal{F}_{c}\{g(x)\}$, we have

$$
\begin{aligned}
\mathcal{F}_{c}^{-1}\left\{F_{c}(k) G_{c}(k)\right\} & =\sqrt{\frac{2}{\pi}} \int_{0}^{\infty} F_{c}(k) G_{c}(k) \cos k x d k \\
& =\frac{2}{\pi} \int_{0}^{\infty} F_{c}(k) \int_{0}^{\infty} g(\xi) \cos k \xi \cos k x d \xi d k .
\end{aligned}
$$


But, $\cos k x \cos k \xi=\frac{1}{2}[\cos k(x-\xi)+\cos k(x+\xi)]$. Thus, substituting and then interchanging the order of integration, we obtain

$$
\begin{aligned}
\mathcal{F}_{c}^{-1}\left\{F_{c}(k) G_{c}(k)\right\} & =\frac{1}{\pi} \int_{0}^{\infty} g(\xi) \int_{0}^{\infty} F_{c}(k)[\cos k(x-\xi)+\cos k(x+\xi)] d k d \xi \\
& =\frac{1}{\sqrt{2 \pi}} \int_{0}^{\infty} g(\xi)\left[f^{(+)}(x-\xi)+f(x+\xi)\right] d \xi,
\end{aligned}
$$

where $f^{(+)}$appears because $(x-\xi)<0$ when $x \leqslant \xi<\infty$.

The same operations performed on the Fourier sine transform requires use of

$$
\sin k x \sin k \xi=\frac{1}{2}[\cos k(x-\xi)-\cos k(x+\xi)]
$$

which leads to

$$
\mathcal{F}_{S}^{-1}\left\{F_{S}(k) G_{s}(k)\right\}=\frac{1}{\pi} \int_{0}^{\infty} g(\xi) \int_{0}^{\infty} F_{s}(k)[\cos k(x-\xi)-\cos k(x+\xi)] d k d \xi .
$$

The sine transform $F_{s}(k)$ is now paired with cosine functions and so does not become inverted. Instead, we have to define a new function $f^{(\sim)}(x)=\mathcal{F}_{c}^{-1}\left\{F_{s}(k)\right\}$ in terms of which the convolution theorem becomes

$$
\mathcal{F}_{s}^{-1}\left\{F_{s}(k) G_{s}(k)\right\}=\frac{1}{\sqrt{2 \pi}} \int_{0}^{\infty} g(\xi)\left[f^{(\sim)}(x-\xi)-f^{(\sim)}(x+\xi)\right] d \xi .
$$

It only remains to explore applications of the three types of Fourier transforms, the most important of which are in the solution of differential equations. However, before we do so we shall introduce another and closely related integral transform, that of Laplace.

\subsection{Laplace Transforms}

Definition: A function $f(x)$ is said to be of exponential order $\sigma$ if $\sigma$ is the largest real number such that $\left|e^{-\sigma x} f(x)\right|$ is bounded on $0 \leqslant x<\infty$. In other words, $f(x)$ does not increase faster than $e^{\sigma x}$ as $x \rightarrow \infty$.

The Fourier transform of a function of non-zero exponential order will not exist because $|f(x)|$ will be unbounded (even in the sense of a polynomial bound) at either $\infty$ or $-\infty$ depending on the sign of $\sigma$. Therefore, for such functions, we form the product

$$
g(x)=f(x) e^{-c x} \theta(x) \text {, where } c>\sigma \text { and } \theta(x)=\left\{\begin{array}{ll}
1, & x>0 \\
0, & x<0
\end{array} .\right.
$$


We are now assured of the convergence of the transform of this function and so define

$$
G(k) \equiv \mathcal{F}\left\{f(x) e^{-c x} \theta(x)\right\}=\frac{1}{\sqrt{2 \pi}} \int_{0}^{\infty} f(x) e^{-c x} e^{i k x} d x
$$

with

$$
\mathcal{F}^{-1}\{G(k)\}=\frac{1}{\sqrt{2 \pi}} \int_{-\infty}^{\infty} G(k) e^{-i k x} d k=f(x) e^{-c x} \theta(x) .
$$

Let us introduce a new transform variable $s=c-i k, d s=-i d k$ and set

$$
F(s)=\sqrt{2 \pi} G(k) .
$$

Then, equations (8.11.1) and (8.11.2) become

$$
\begin{gathered}
F(s)=\int_{0}^{\infty} f(t) e^{-s t} d t \equiv \mathcal{L}\{f(t)\} \\
f(t) \theta(t)=\frac{1}{2 \pi i} \int_{c-i \infty}^{c+i \infty} F(s) e^{s t} d s \equiv \mathcal{L}^{-1}\{F(s)\}
\end{gathered}
$$

where, to conform with convention, we have replaced the symbol $x$ for the independent variable with the letter $t$. These are the defining equations for the Laplace transform and its inverse. (The integral in (8.11.4) is often referred to as the Mellin inversion integral.) Evidently, the Laplace transform offers a means of extending the applicability of Fourier transforms to functions for which the Fourier integral is not defined. As such, they are widely used in the solution of engineering problems.

Not surprisingly, the properties of Laplace transforms are close analogs of those of Fourier transforms. Specifically, there is

- an attenuation property,

$$
\mathcal{L}\left\{e^{-a t} f(t)\right\}=F(s+a) \text { where } F(s)=\mathcal{L}\{f(t)\} ;
$$

- a shifting property,

$$
\mathcal{L}\{f(t-a) \theta(t-a)\}=e^{-a s} \mathcal{L}\{f(t)\}, \quad a>0 ;
$$

- the derivative property,

$$
\mathcal{L}\left\{f^{\prime}(t)\right\}=s \mathcal{L}\{f(t)\}-f(0)
$$

which extends to

$$
\mathcal{L}\left\{f^{\prime \prime}(t)\right\}=s \mathcal{L}\left\{f^{\prime}(t)\right\}-f^{\prime}(0)=s^{2} \mathcal{L}\{f(t)\}-s f(0)-f^{\prime}(0),
$$


and by induction to

$$
\mathcal{L}\left\{f^{(n)}(t)\right\}=s^{n} \mathcal{L}\{f(t)\}-\sum_{k=1}^{n} s^{k-1} f^{(n-k)}(0),
$$

and is readily established by a single integration by parts,

$$
\begin{gathered}
\int_{0}^{\infty} e^{-s t} f(t) d t=-\left.\frac{1}{s} e^{-s t} f(t)\right|_{0} ^{\infty}+\int_{0}^{\infty} e^{-s t} f^{\prime}(t) d t \\
\text { or } s \int_{0}^{\infty} e^{-s t} f(t) d t=f(0)+\int_{0}^{\infty} e^{-s t} f^{\prime}(t) d t
\end{gathered}
$$

- multiplication by a power of $t$ property,

$$
\begin{array}{r}
\mathcal{L}\{t f(t)\}=-\frac{d}{d s} \mathcal{L}\{f(t)\} \\
\mathcal{L}\left\{t^{n} f(t)\right\}=(-1)^{n} \frac{d^{n}}{d s^{n}} \mathcal{L}\{f(t)\} ;
\end{array}
$$

- and the convolution theorem,

$$
\mathcal{L}^{-1}\{F(s) G(s)\}=\int_{0}^{t} f(\tau) g(t-\tau) d \tau
$$

if $F(s)=\mathcal{L}\{f(t)\}$ and $G(s)=\mathcal{L}\{g(t)\}$.

The most important application of Laplace transforms is in the solution of differential equations, especially linear differential equations. As with the use of Fourier transforms, the method consists of transforming a given differential equation to yield a subsidiary equation which, if the choice of method is appropriate, is an easier equation to solve. In fact, if the differential equation is linear, the subsidiary equation is algebraic and so is solvable by purely algebraic techniques. The choice of Laplace rather than some variety of Fourier transform hinges on the boundary conditions associated with the differential equation. Specifically, Laplace transforms are appropriate to boundaries at $t=0$ and $\infty$ with a knowledge of the values of the solution and its first derivative at the first of these. The final step in this method is to invert the transform obtained as a solution of the subsidiary equation. For Laplace transforms this can be done by using the Mellin inversion integral. More commonly, however, one makes use of a knowledge of a few key Laplace transform pairs sufficient to invert any rational function of $s$, or if the solution of the subsidiary equation is more complicated, one consults a comprehensive table of Laplace transform pairs.

The inversion of a rational function proceeds as follows. Let the function be $Y(s)=$ $P(s) / Q(s)$ where $P(s)$ and $Q(s)$ are polynomials with $\operatorname{deg}(P)>\operatorname{deg}(Q)$. If $Q(s)$ has $n$ 
simple real roots $r_{i}, i=1,2, \ldots, n$, we can express $Y(s)$ in terms of the corresponding partial fractions:

$$
Y(s)=\sum_{i=1}^{n} \frac{c_{i}}{s-r_{i}}+W(s)
$$

where $W(s)$ is the sum of partial fractions associated with all the other roots of $Q(s)$. The constants $c_{i}$ can be found by multiplying both sides of this equation by $\left(s-r_{i}\right)$ and taking the limit as $s \rightarrow r_{i}$ :

$$
c_{i}=\lim _{s \rightarrow r_{i}}\left(s-r_{i}\right) Y(s)=\frac{P\left(r_{i}\right)}{Q^{\prime}\left(r_{i}\right)} .
$$

Thus, using $\mathcal{L}\left\{e^{r_{i} t}\right\}=\frac{1}{s-r_{i}}$, we have

$$
y(t)=\mathcal{L}^{-1}\{Y(s)\}=\sum_{i=1}^{n} \frac{P\left(r_{i}\right)}{Q^{\prime}\left(r_{i}\right)} e^{r_{i} t}+\mathcal{L}^{-1}\{W(s)\} .
$$

Example: Suppose that we seek $\mathcal{L}^{-1}\left\{\frac{s+1}{s^{3}+s^{2}-6 s}\right\}$. In terms of partial fractions, we have

$$
\frac{s+1}{s^{3}+s^{2}-6 s}=\frac{s+1}{s(s-2)(s+3)}=\frac{c_{1}}{s}+\frac{c_{2}}{s-2}+\frac{c_{3}}{s+3}
$$

where

$$
\begin{aligned}
& c_{1}=P(0) / Q^{\prime}(0)=\frac{1}{\left.3 s^{2}+2 s-6\right)\left.\right|_{s=0}}=-\frac{1}{6} \\
& c_{2}=P(2) / Q^{\prime}(2)=\frac{2}{3 \cdot 4+4-6}=\frac{3}{10} \\
& c_{3}=P(-3) / Q^{\prime}(-3)=\frac{-2}{3 \cdot 9-6-6}=-\frac{2}{15} .
\end{aligned}
$$

Therefore,

$$
y(t)=-\frac{1}{6}+\frac{3}{10} e^{2 t}-\frac{2}{15} e^{-3 t} .
$$

If $Q(s)$ has a real root $r$ of multiplicity $m, Y(s)$ will have a partial fraction decomposition of the form

$$
Y(s)=\frac{c_{m}}{(s-r)^{m}}+\frac{c_{m-1}}{(s-r)^{m-1}}+\ldots+\frac{c_{1}}{s-r}+W(s)
$$

where $W(s)$ again denotes the sum of the partial fractions associated with the other roots of $Q(s)$. To determine $c_{m}$ we multiply both sides of this equation by $(s-r)^{m}$ :

$$
G(s) \equiv(s-r)^{m} Y(s)=c_{m}+(s-r) c_{m-1}+\ldots+(s-r)^{m-1} c_{1}+(s-r)^{m} W(s) .
$$

Setting $s=r$, we obtain

$$
c_{m}=G(r) .
$$


To determine $c_{m-1}$ we differentiate $G(s)$,

$$
G^{\prime}(s)=c_{m-1}+2(s-r) c_{m-2}+\ldots+m(s-r)^{m-1} W(s)+(s-r)^{m} W^{\prime}(s),
$$

and set $s=r$ to obtain

$$
c_{m-1}=G^{\prime}(r) \text {. }
$$

Continuing in this fashion up to and including the $(m-1)^{\text {th }}$ derivative, we find

$$
c_{m-2}=\frac{1}{2 !} G^{\prime \prime}(r), \ldots, c_{k}=\frac{1}{(m-k) !} G^{(m-k)}(r), \ldots, c_{1}=\frac{1}{(m-1) !} G^{(m-1)}(r) .
$$

Now, $\mathcal{L}^{-1}\left\{\frac{1}{s^{k}}\right\}=\frac{t^{k-1}}{(k-1) !}$ and so, by the attenuation property,

$$
\mathcal{L}^{-1}\left\{\frac{1}{(s-r)^{k}}\right\}=\frac{e^{r t} t^{k-1}}{(k-1) !}
$$

Therefore,

$$
\begin{aligned}
y(t)=\mathcal{L}^{-1}\{Y(s)\} & =\left[\frac{c_{m} t^{m-1}}{(m-1) !}+\frac{c_{m-1} t^{m-2}}{(m-2) !}+\ldots+c_{2} t+c_{1}\right] e^{r t}+\mathcal{L}^{-1}\{W(s)\} \\
& =e^{r t} \sum_{k=1}^{m} \frac{G^{(m-k)}(r)}{(k-1) !(m-k) !} t^{k-1}+\mathcal{L}^{-1}\{W(s)\} .
\end{aligned}
$$

Example: Suppose that we wish to invert $\frac{s+2}{s^{5}-2 s^{4}+s^{3}}$. This rational function has the partial fraction decomposition

$$
\frac{s+2}{s^{5}-2 s^{4}+s^{3}}=\frac{a_{3}}{s^{3}}+\frac{a_{2}}{s^{2}}+\frac{a_{1}}{s}+\frac{b_{2}}{(s-1)^{2}}+\frac{b_{1}}{s-1} .
$$

To determine the coefficients $a_{i}$, we define $G(s)=s^{3} Y(s)=\frac{s+2}{(s-1)^{2}}$. Then,

$$
a_{3}=G(0)=2, \quad a_{2}=G^{\prime}(0)=5, \quad a_{3}=\frac{G^{\prime \prime}(0)}{2 !}=8 .
$$

To determine the $b_{i}$, define $H(s)=(s-1)^{2} Y(s)=\frac{s+2}{s^{3}}$. Then,

$$
b_{2}=H(1)=3 \text { and } b_{1}=H^{\prime}(1)=-8 \text {. }
$$

Thus,

$$
y(t)=\mathcal{L}^{-1}\{Y(s)\}=2 t^{2}+5 t+8+e^{t}(3 t-8) .
$$

If $Q(s)$ has real coefficients, any complex roots it may have will occur in conjugate pairs: $r=\alpha+i \beta$ and $r^{\star}=\alpha-i \beta$. If $P(s)$ also has real coefficients, the partial fractions associated with these roots will have complex conjugate coefficients since

$$
\frac{P\left(r^{\star}\right)}{Q^{\prime}\left(r^{\star}\right)}=\left[\frac{P(r)}{Q^{\prime}(r)}\right]^{\star}=c^{\star} \text {. }
$$


Thus, using $e^{r t}=e^{\alpha t}[\cos \beta t+i \sin \beta t]$ and equation (8.11.12), we deduce for the case of $n$ pairs of simple complex roots that

$$
y(t)=2 \sum_{j=1}^{n} e^{\alpha_{j} t}\left[\operatorname{Re}\left(\frac{P\left(r_{j}\right)}{Q^{\prime}\left(r_{j}\right)}\right) \cos \beta_{j} t-\operatorname{Im}\left(\frac{P\left(r_{j}\right)}{Q^{\prime}\left(r_{j}\right)}\right) \sin \beta_{j} t\right]+\mathcal{L}^{-1}\{W(s)\}
$$

where $r_{j}=\alpha_{j}+i \beta_{j}$ is a member of the $j^{t h}$ pair.

For a pair of roots with multiplicity $m$, we invoke (8.11.13) rather than (8.11.12) and obtain

$$
y(t)=\sum_{k=1}^{m} \frac{2 t^{k-1}}{(k-1) !} e^{\alpha t}\left[\operatorname{Re} a_{k} \cos \beta t-\operatorname{Im} a_{k} \sin \beta t\right]+\mathcal{L}^{-1}\{W(s)\}
$$

where $a_{k}=\frac{1}{(m-1) !} G^{(m-k)}(r), G(s)=(s-r)^{m} Y(s)$, and $r=\alpha+i \beta$.

Example: Consider the rational function $Y(s)=\frac{2 s}{s^{2}+2 s+5}$. Its denominator has roots at $s=-1 \pm 2 i$. Thus, setting $r=\alpha+i \beta=-1+2 i$, we have

$$
\left.\frac{P(s)}{Q^{\prime}(s)}\right|_{s=r}=\left.\frac{s}{s+1}\right|_{s=r}=\frac{-1+2 i}{2 i}=1+\frac{i}{2}
$$

and

$$
y(t)=e^{-t}(2 \cos 2 t-\sin 2 t)
$$

\subsection{Application: Solving Differential Equations}

One of the most important applications of integral transfoms is in the solution of boundary value problems. These are problems that seek the one solution of a given differential equation that satisfies certain conditions at the boundaries of the interval of variation of the independent variable. The conditions can involve specification of the value of the solution, of its first derivative, or of some linear combination of the two.

Because of the simple form assumed by their respective derivative properties, (which is due to the exponential nature of their kernels), Fourier and Laplace transforms are best suited to problems involving a differential equation with constant coefficients. Such equations become transformed into subsidiary equations that contain no derivatives and admit simple algebraic solutions. The latter are then subjected to an inverse transformation to produce the solutions that are appropriate to the problems in which the differential equations occur.

Since each transform requires a unique set of input or boundary condition information, one must be careful in the selection of one to use in the solution of a particular problem. It is not sufficient that the problem involves a differential equation with constant coefficients, it must have the correct range, $[0, \infty)$ or $(-\infty, \infty)$, for the independent variable and must contain boundary conditions that match the input needs 
of either Laplace, Fourier sine, Fourier cosine or Fourier transforms. These are summarized in the Table below (Table 8.1).

To illustrate these points we begin with the application of the Laplace transformation to the linear differential equation

$$
y^{\prime \prime}(t)+a y^{\prime}(t)+b y(t)=r(t)
$$

where $a$ and $b$ are known constants and $r(t)$ is a known function. Using the derivative property, equations (8.11.7) and (8.11.8), we obtain the subsidiary equation

$$
s^{2} Y(s)-s y(0)-y^{\prime}(0)+a(s Y(s)-y(0))+b Y(s)=R(s)
$$

where $Y(s)=\mathcal{L}\{y(t)\}$ and $R(s)=\mathcal{L}\{r(t)\}$. Its solution is

$$
Y(s)=\frac{(s+a) y(0)+y^{\prime}(0)}{s^{2}+a s+b}+\frac{R(s)}{s^{2}+a s+b} .
$$

Table 8.1: Applicability Conditions of Integral Transforms

\begin{tabular}{ccc}
\hline Transformation & Range of Independent Variable & Boundary Conditions \\
\hline Fourier & $-\infty<x<\infty$ & $y( \pm \infty)=y^{\prime}( \pm \infty)=0$ \\
Fourier sine & $0 \leqslant x<\infty$ & $y(0)=c_{1}, y(\infty)=y^{\prime}(\infty)=0$ \\
Fourier cosine & $0 \leqslant x<\infty$ & $y^{\prime}(0)=c_{2}, y(\infty)=y^{\prime}(\infty)=0$ \\
Laplace & $0 \leqslant t<\infty$ & $y(0)=c_{1}, y^{\prime}(0)=c_{2}$ \\
\hline
\end{tabular}

The next and final step is to determine the inverse transform $\mathcal{L}^{-1}\{Y(s)\}=y(t)$ and thus obtain the solution of the differential equation. The inverse of the first term in our expression for $Y(s)$ is a solution of the corresponding homogeneous equation and is called the complementary function. It matches the boundary conditions (or initial conditions if $t$ is a time variable) $y(0)=c_{1}$ and $y^{\prime}(0)=c_{2}$ that have to accompany the differential equation if this method is to be useful. The inverse of the second term yields a particular solution of the non-homogeneous equation corresponding to the conditions $y(0)=y^{\prime}(0)=0$. It is called a particular integral. Note that the first term as well as $\frac{1}{s^{2}+a s+b}$ are rational functions. They can be inverted by means of the techniques identified at the end of the last Section. The second term can then be inverted by application of the convolution theorem.

Examples: We shall start by paying a return visit to the forced, damped harmonic oscillator

$$
m \frac{d^{2} x}{d t^{2}}+r \frac{d x}{d t}+k x=f(t), \quad 0 \leqslant t<\infty
$$

or,

$$
\frac{d^{2} x}{d t^{2}}+2 \lambda \frac{d x}{d t}+\omega_{0}^{2} x=\frac{1}{m} f(t) \text { where } \lambda=\frac{r}{2 m} \text { and } \omega_{0}^{2}=\frac{k}{m} .
$$

If the initial conditions specify both $x(0)$ and $x^{\prime}(0), x(0)=x_{0}$ and $x^{\prime}(0)=v_{0}$ for example, it is appropriate to use Laplace transforms to solve for the motion of the 
oscillator. The solution of the resulting subsidiary equation is

$$
X(s)=\frac{2 \lambda x_{0}+v_{0}+s x_{0}}{s^{2}+2 \lambda s+\omega_{0}^{2}}+\frac{F(s)}{s^{2}+2 \lambda s+\omega_{0}^{2}}=X_{c}(s)+X_{p}(s) .
$$

The first term can be expressed as

$$
X_{c}(s)=\frac{x_{0}(s+\lambda)+\left(v_{0}+\lambda x_{0}\right)}{(s+\lambda)^{2}+\left(\omega_{0}^{2}-\lambda^{2}\right)}
$$

Assuming that $\omega_{0}^{2}>\lambda^{2}$, which is usually the case, this has the inverse transform

$$
x_{c}(t)=x_{0} e^{-\lambda t} \cos \sigma t+\frac{v_{0}+\lambda x_{0}}{\sigma} e^{-\lambda t} \sin \sigma t, \quad \sigma=\sqrt{\omega_{0}^{2}-\lambda^{2}} .
$$

This is the complementary function, or solution of the homogeneous differential equation, that satisfies the initial conditions that have been imposed in this problem.

To invert $X_{p}(s)$, we note that

$$
\mathcal{L}^{-1}\{F(s)\}=f(t) \text { and } \mathcal{L}^{-1}\left\{\frac{1}{s^{2}+2 \lambda s+\omega_{0}^{2}}\right\}=\frac{1}{\sigma} e^{-\lambda t} \sin \sigma t, \sigma=\sqrt{\omega_{0}^{2}-\lambda^{2}} .
$$

Thus, using the convolution theorem, we can write

$$
x_{p}(t)=\mathcal{L}^{-1}\left\{\frac{F(s)}{s^{2}+2 \lambda s+\omega_{0}^{2}}\right\}=\int_{0}^{t} \frac{1}{\sigma} e^{-\lambda(t-\tau)} \sin \sigma(t-\tau) f(\tau) d \tau
$$

so that the complete solution to our problem reads

$$
x(t)=x_{c}(t)+x_{p}(t)=x_{0} e^{-\lambda t} \cos \sigma t+\frac{v_{0}+\lambda x_{0}}{\sigma} e^{-\lambda t} \sin \sigma t+\frac{1}{m \sigma} \int_{0}^{t} e^{-\lambda(t-\tau)} \sin \sigma(t-\tau) f(\tau) d \tau .
$$

To illustrate what happens at resonance, let us assume for simplicity that the damping is negligible so that $\lambda \approx 0$ and $\sigma \approx \omega_{0}$. Then, if $f(t)=K \sin \omega_{0} t$, where $K$ is a constant, we have

$$
x(t)=x_{0} \cos \omega_{0} t+\frac{v_{0}}{\omega_{0}} \sin \omega_{0} t+\frac{K}{m \omega_{0}} \int_{0}^{t} \sin \omega_{0}(t-\tau) \sin \omega_{0} \tau d \tau .
$$

But,

$$
\begin{aligned}
\int_{0}^{t} \sin \omega_{0} \tau \sin \omega_{0}(t-\tau) d \tau & =\sin \omega_{0} t \int_{0}^{t} \sin \omega_{0} \tau \cos \omega_{0} \tau d \tau-\cos \omega_{0} t \int_{0}^{t} \sin ^{2} \omega_{0} \tau d \tau \\
& =\frac{1}{4 \omega_{0}}\left[\sin \omega_{0} t\left(1-\cos 2 \omega_{0} t\right)-\cos \omega_{0} t\left(2 \omega_{0} t-\sin 2 \omega_{0} t\right)\right] \\
& =\frac{1}{2 \omega_{0}}\left(\sin \omega_{0} t-\omega_{0} t \cos \omega_{0} t\right) .
\end{aligned}
$$


This means that the last term in the particular integral

$$
x_{p}(t)=\frac{K}{2 m \omega_{0}^{2}}\left(\sin \omega_{0} t-\omega_{0} t \cos \omega_{0} t\right)
$$

has an amplitude that increases linearly with $t$ corresponding to a resonant response by the system. The resonance is due, of course, to the frequency of the applied force coinciding with the natural frequency of the oscillator.

Suppose that instead of an harmonic force, the oscillator is subjected to an instantaneous impulse of magnitude $I$ at time $t=t_{0}$. The force responsible for the impulse must be expressible as $f(t)=I \delta\left(t-t_{0}\right)$. Rather than use the convolution theorem, we note that the transform of $f(t)$ is particularly simple: $\mathcal{L}\{f(t)\}=e^{-s t_{0}}$. Thus,

$$
X_{p}(s)=\frac{I}{m} \frac{e^{-s t_{0}}}{s^{2}+2 \lambda s+\omega_{0}^{2}} .
$$

But, according to equation (8.10.6), (the shifting property of Laplace transforms), the inverse of this product is just $\mathcal{L}^{-1}\left\{e^{s t_{0}} X_{p}(s)\right\} \cdot \theta(t)$ with $t$ replaced by $t-t_{0}$ :

$$
\mathcal{L}^{-1}\left\{X_{p}(s)\right\}=x_{p}(t)=\frac{I}{m \sigma} e^{-\lambda\left(t-t_{0}\right)} \sin \sigma\left(t-t_{0}\right) \theta\left(t-t_{0}\right) .
$$

Therefore, the motion of the oscillator is given by

$$
x(t)=x_{0} e^{-\lambda t} \cos \sigma t+\frac{v_{0}+\lambda x_{0}}{\sigma} e^{-\lambda t} \sin \sigma t+\frac{I}{m \sigma} e^{-\lambda\left(t-t_{0}\right)} \sin \sigma\left(t-t_{0}\right) \theta\left(t-t_{0}\right)
$$

demonstrating explicitly that whatever motion is initiated at $t=0$ ( by assignment of values to $x_{0}$ and $v_{0}$ ) it is modified at $t=t_{0}$ and thereafter by the motion caused by the impulse.

Another example along the same line is provided by an LRC-series consisting of an inductance $\mathrm{L}$, resistance $\mathrm{R}$ and capacitance $\mathrm{C}$ connected in series to a switch and an emf $e(t)$. The switch is closed from $t=0$ to $t=T$. We seek the current $i(t)$ in the circuit assuming that it is zero at $t=0$ along with the charge $q(t)$ on the capacitor: $i(0)=0$ and $q(0)=0$.

The current is governed by Kirchoff's Law which requires that

$$
L \frac{d i(t)}{d t}+R i(t)+\frac{q(t)}{C}=e(t)
$$

where we shall assume

$$
e(t)=\left\{\begin{array}{cl}
e_{0}, & 0<t<T \\
0, & t>T .
\end{array}\right.
$$

Moreover, we know that $i(t)=\frac{d q(t)}{d t}$.

Applying the Laplace transform to these two differential equations we obtain

$$
L s I(s)-L i(0)+\frac{1}{C} Q(s)=E(s), \quad I(s)=s Q(s)-q(0) .
$$


Thus, using our initial conditions and solving for $I(s)$, we find

$$
I(s)=\frac{E(s)}{L s+R+1 /(s C)}=\frac{1}{L} \frac{s E(s)}{s^{2}+(R / L) s+1 /(L C)}
$$

where

$$
E(s)=e_{0} \int_{0}^{T} e^{-s t} d t=e_{0} \frac{1-e^{-s T}}{s} .
$$

Therefore,

$$
I(s)=\frac{e_{0}}{L} \frac{1-e^{-s T}}{s^{2}+(R / L) s+1 /(L C)} .
$$

The shifting property of Laplace transforms (equation (8.11.6)) takes care of the factor $e^{-s T}$ in the numerator of the expression for $I(s)$ and so all that remains is to find the inverse of $\left\{s^{2}+(R / L) s+1 /(L C)\right\}^{-1}$. Three cases arise depending on the roots of this quadratic and to classify them, we introduce the constants $\alpha=R / 2 L$ and $\omega^{2}=$ $1 / L C-R^{2} / 4 L^{2}$. Then, if $\omega^{2}>0$, the roots of the quadratic are complex,

$$
\mathcal{L}^{-1}\left\{\frac{1}{s^{2}+(R / L) s+1 / L C}\right\}=\frac{1}{\omega} e^{-\alpha t} \sin \omega t
$$

and

$$
i(t)=\frac{e_{0}}{\omega L} e^{-\alpha t} \sin \omega t-\frac{e_{0}}{\omega L} e^{-\alpha(t-T)} \sin \omega(t-T) \theta(t-T) .
$$

This is called the oscillatory case.

If $0>\omega^{2}=-\beta^{2}$, the roots are real and, replacing $\omega$ by $i \beta$, we have

$$
i(t)=\frac{e_{0}}{\beta L} e^{-\alpha t} \sinh \beta t-\frac{e_{0}}{\beta L} e^{-\alpha(t-T)} \sinh \beta(t-T) \theta(t-T) .
$$

This is called the overdamped case.

Finally, if $\omega^{2}=0$, there is a double root and so

$$
i(t)=\frac{e_{0}}{L} t e^{-\alpha t}-\frac{e_{0}}{L}(t-T) e^{-\alpha(t-T)} \theta(t-T) .
$$

This is called the critically damped case.

The next example involves a differential equation with variable coefficients and reveals some of the limitations of the Laplace transform method of solution. The differential equation is (Bessel's equation of order zero)

$$
x y^{\prime \prime}+y^{\prime}+x y=0
$$

and the boundary conditions that we wish to impose are $y(0)=1, y^{\prime}(0)=0$.

If $Y(s)=\mathcal{L}\{y(x)\}$, equation (8.11.10) tells us that $\mathcal{L}\{x y(x)\}=-\frac{d Y(s)}{d s}$. Notice that if the coefficient of $y(x)$ were $x^{2}$, we would obtain the second derivative of $Y(s)$ and the subsidiary equation would be another second order differential equation thus doing little to advance the solution of the equation we started with. 
Using the boundary conditions together with the derivative property, we also have

$$
\begin{aligned}
\mathcal{L}\left\{y^{\prime}(x)\right\} & =s Y(s)-1 \\
\mathcal{L}\left\{y^{\prime \prime}(x)\right\} & =s^{2} Y(s)-s .
\end{aligned}
$$

Thus, invoking (8.11.10) again, we obtain

$$
\mathcal{L}\left\{x^{2} y(x)\right\}=-2 s Y(s)-s^{2} \frac{d Y(s)}{d s}+1
$$

and so the subsidiary equation is

$$
-2 s Y(s)-s^{2} Y^{\prime}(s)+1+s Y(s)-1-Y^{\prime}(s)=0,
$$

or

$$
\left(s^{2}+1\right) Y^{\prime}(s)+s Y(s)=0 .
$$

The solution of this differential equation is easy to find:

$$
\ln Y(s)=-\int \frac{s}{s^{2}+1} d s=-\frac{1}{2} \ln \left(s^{2}+1\right)+\text { cnst. }
$$

and so, $Y(s)=\frac{c}{\sqrt{s^{2}+1}}$ where $c$ is a constant.

To find $y(x)$, we expand $Y(s)$ in inverse powers of $s$ (a Laurent series valid for $|s|>$ 1):

$$
Y(s)=\frac{c}{s}\left(1+\frac{1}{s^{2}}\right)^{-1 / 2}=c \sum_{n=0}^{\infty} \frac{(-1)^{n}(2 n) !}{2^{2 n}(n !)^{2}} \frac{1}{s^{2 n+1}} .
$$

Inverting term be term, we find

$$
y(x)=c \sum_{n=0}^{\infty} \frac{(-1)^{n} x^{2 n}}{2^{2 n}(n !)^{2}} \text { since } \mathcal{L}^{-1}\left\{\frac{1}{s^{2 n+1}}\right\}=\frac{x^{2 n}}{(2 n) !} .
$$

But $y(0)=1$. Therefore, $c=1$, and our solution becomes

$$
y(x)=\sum_{n=0}^{\infty} \frac{(-1)^{n}}{(n !)^{2}}\left(\frac{x}{2}\right)^{2 n}
$$

which is called the Bessel function of order zero and is conventionally denoted by $J_{0}(x)$.

As we shall see in the next Chapter, this differential equation has a second linearly independent solution which has an essential singularity at $x=0$. Since the Laplace transform method requires well-defined values for both $y(0)$ and $y^{\prime}(0)$, it is useless if that is the solution we seek.

To furnish examples of the application of Fourier transforms to the solution of differential equations, we return to the problem of an harmonic oscillator acted on by an external force. Using the same notation as before, the equation of motion is

$$
\frac{d^{2} x}{d t^{2}}+2 \lambda \frac{d x}{d t}+\omega_{0}^{2} x=\frac{1}{m} f(t)
$$


where $t$ is presumed now to have the range $-\infty<t<\infty$. If we presume also that $x(t) \rightarrow 0$ as $t \rightarrow \pm \infty$ so that it possesses a Fourier transform, we can transform the differential equation to obtain the subsidiary equation

$$
-\omega^{2} X(\omega)-2 \lambda \omega i X(\omega)+\omega_{0}^{2} X(\omega)=\frac{1}{m} F(\omega)
$$

where $X(\omega)=\mathcal{F}\{x(t)\}$ and $F(\omega)=\mathcal{F}\{f(t)\}$. This has the solution

$$
X(\omega)=\frac{1}{m} \frac{F(\omega)}{\left(\omega_{0}^{2}-\omega^{2}\right)-2 \lambda \omega i}
$$

and so the solution of the original problem is

$$
x(t)=\frac{1}{\sqrt{2 \pi}} \int_{-\infty}^{\infty} \frac{1}{m} \frac{F(\omega) e^{-i \omega t}}{\left(\omega_{0}^{2}-\omega^{2}\right)-2 \lambda \omega i} d \omega .
$$

In most cases this integral can be evaluated by residue calculus. To illustrate how, we shall take $\omega_{0}>\lambda$, which corresponds to a weakly damped oscillator, and assume a force of the form

$$
f(t)=\left\{\begin{array}{cc}
f_{0}, & |t|<\tau \\
0, & |t| \geqslant \tau
\end{array}\right.
$$

We then have

$$
F(\omega)=\frac{f_{0}}{\sqrt{2 \pi}} \int_{-\tau}^{\tau} e^{i \omega t} d t=f_{0} \sqrt{\frac{2}{\pi}} \frac{\sin \omega t}{\omega}
$$

and so,

$$
x(t)=-\frac{f_{0}}{m \pi} \int_{-\infty}^{\infty} \frac{\sin \omega \tau e^{-i \omega t}}{\omega\left(\omega-\omega_{1}\right)\left(\omega-\omega_{2}\right)} d \omega
$$

where $\omega_{1}=\sigma-\lambda i, \omega_{2}=-\sigma-\lambda i, \sigma=\sqrt{\omega_{0}^{2}-\lambda^{2}}$.

Expressing $\sin \omega \tau$ in terms of exponentials and deforming the contour to avoid introducing an extraneous singularity at $\omega=0$, we can rewrite $x(t)$ as

$$
x(t)=-\frac{f_{0}}{m 2 \pi i} \int_{-\cup \rightarrow} \frac{e^{-i \omega(t-\tau)}}{\omega\left(\omega-\omega_{1}\right)\left(\omega-\omega_{2}\right)} d \omega+\frac{f_{0}}{m 2 \pi i} \int_{-\cup \rightarrow} \frac{e^{-i \omega(t+\tau)}}{\omega\left(\omega-\omega_{1}\right)\left(\omega-\omega_{2}\right)} d \omega
$$

where the subscript on the integral signs indicates that we are going below the real axis in the neighbourhood of $\omega=0$. We shall evaluate each term separately beginning with the first.

If $t-\tau>0$, we must close the contour of the first integral in the lower half plane to be able to use the residue theorem. If $t-\tau<0$, we close in the upper half plane. Thus,

$$
\text { the first term }=\left\{\begin{array}{cc}
\frac{f_{0}}{m} \frac{e^{-i \omega_{1}(t-\tau)}}{\omega_{1}\left(\omega_{1}-\omega_{2}\right)}-\frac{f_{0}}{m} \frac{e^{-i \omega_{2}(t-\tau)}}{\omega_{2}\left(\omega_{1}-\omega_{2}\right)}, & t>\tau \\
-\frac{f_{0}}{m} \frac{1}{\omega_{1} \omega_{2}}, & t<\tau .
\end{array}\right.
$$


Similarly, the second integral's contour must be closed in the lower half-plane if $t+\tau>0$ and in the upper half-plane if $t+\tau<0$. Thus,

$$
\text { the second term }=\left\{\begin{array}{cl}
-\frac{f_{0}}{m} \frac{e^{-i \omega_{1}(t+\tau)}}{\omega_{1}\left(\omega_{1}-\omega_{2}\right)}+\frac{f_{0}}{m} \frac{e^{-i \omega_{2}(t+\tau)}}{\omega_{2}\left(\omega_{1}-\omega_{2}\right)}, & t>-\tau \\
\frac{f_{0}}{m} \frac{1}{\omega_{1} \omega_{2}}, & t<-\tau .
\end{array}\right.
$$

This provides us with three cases:

1. when $t<-\tau$, we have

$$
x(t)=-\frac{f_{0}}{m} \frac{1}{\omega_{1} \omega_{2}}+\frac{f_{0}}{m} \frac{1}{\omega_{1} \omega_{2}}=0
$$

which confirms that the damped oscillator is at rest until subjected to the external force;

2. when $-\tau<t<\tau$, the displacement is

$$
\begin{aligned}
x(t) & =-\frac{f_{0}}{m} \frac{1}{\omega_{1} \omega_{2}}-\frac{f_{0}}{m} \frac{e^{-i \omega_{1}(t+\tau)}}{\omega_{1}\left(\omega_{1}-\omega_{2}\right)}+\frac{f_{0}}{m} \frac{e^{-i \omega_{2}(t+\tau)}}{\omega_{2}\left(\omega_{1}-\omega_{2}\right)} \\
& =\frac{f_{0}}{m \omega_{0}^{2}}-\frac{f_{0}}{m \omega_{0}^{2}}\left[\cos \sigma(t+\tau)+\frac{\lambda}{\sigma} \sin \sigma(t+\tau)\right] e^{-\sigma(t+\tau)}
\end{aligned}
$$

3. and when $t>\tau$, it is

$$
\begin{aligned}
x(t)= & \frac{f_{0}}{m} \frac{e^{-i \omega_{1}(t-\tau)}}{\omega_{1}\left(\omega_{1}-\omega_{2}\right)}-\frac{f_{0}}{m} \frac{e^{-i \omega_{2}(t-\tau)}}{\omega_{2}\left(\omega_{1}-\omega_{2}\right)}-\frac{f_{0}}{m} \frac{e^{-i \omega_{1}(t+\tau)}}{\omega_{1}\left(\omega_{1}-\omega_{2}\right)}+\frac{f_{0}}{m} \frac{e^{-i \omega_{2}(t+\tau)}}{\omega_{2}\left(\omega_{1}-\omega_{2}\right)} \\
= & \frac{f_{0}}{m \omega_{0}^{2}}\left[\cos \sigma(t-\tau)+\frac{\lambda}{\sigma} \sin \sigma(t-\tau)\right] e^{-\lambda(t-\tau)} \\
& -\frac{f_{0}}{m \omega_{0}^{2}}\left[\cos \sigma(t+\tau)+\frac{\lambda}{\sigma} \sin \sigma(t+\tau)\right] e^{-\lambda(t+\tau)} .
\end{aligned}
$$

A rather special problem arises in the (physically unlikely) event that there is no damping. The equation of motion of the oscillator becomes

$$
\frac{d^{2} x}{d t^{2}}+\omega_{0}^{2} x=\frac{f(t)}{m}
$$

which, when solved by the Fourier transform procedure, yields a solution of the form

$$
x(t)=\frac{1}{m \sqrt{2 \pi}} \int_{-\infty}^{\infty} \frac{F(\omega) e^{-i \omega t}}{\omega_{0}^{2}-\omega^{2}} d \omega .
$$

The poles arising from the zeros of the denominator are now on the real axis at $\omega=$ $\pm \omega_{0}$ and so the integral is undefined until we specify how we propose to avoid them.

Additional physical information is needed to resolve this ambiguity. For example, suppose that the oscillator is at rest until disturbed by a sharp blow delivered at $t=t_{0}$. 
Representing the external force by means of a $\delta$-function, $f(t)=f_{0} \delta\left(t-t_{0}\right)$ where $f_{0}$ is a constant, we have

$$
F(\omega)=\frac{1}{\sqrt{2 \pi}} \int_{-\infty}^{\infty} f_{0} \delta\left(t-t_{0}\right) e^{i \omega t} d t=\frac{f_{0}}{\sqrt{2 \pi}} e^{i \omega t_{0}}
$$

and,

$$
x(t)=\frac{f_{0}}{m} \frac{1}{2 \pi} \int_{-\infty}^{\infty} \frac{e^{-i \omega\left(t-t_{0}\right)}}{\omega_{0}^{2}-\omega^{2}} d \omega .
$$

Now, when $t-t_{0}<0$, this expression should yield the value $x(t)=0$, since otherwise we would have motion occurring prior to the impulse in violation of our initial assumption and of the principle of causality. Moreover, when $t-t_{0}<0$, Jordan's Lemma permits us to close the contour by means of a semi-circular arc of infinite radius in the upper half-plane and evaluate the integral by means of the Residue Theorem. But the residues at the two poles $\omega= \pm \omega_{0}$ of this integrand are

$$
-\frac{f_{0}}{m} \frac{e^{-i \omega_{0}\left(t-t_{0}\right)}}{4 \pi \omega_{0}}
$$

and

$$
\frac{f_{0}}{m} \frac{e^{+i \omega_{0}\left(t-t_{0}\right)}}{4 \pi \omega_{0}},
$$

respectively. Thus, if one or both of the poles is included within the closed contour, the result will not be zero. We conclude therefore that the integral must be defined by deforming the contour along the real axis to pass above the poles $\omega= \pm \omega_{0}$ as shown in the diagram below. (We note that this is the equivalent of adding a vanishingly small damping force since the latter would shift the poles to $\omega= \pm \omega_{0}-i \varepsilon$.) In other words, the mathematical ambiguity has been resolved by appeal to a fundamental physical principle; we now have an unambiguous definition of the integral and thence, can determine the oscillator's motion to be (by closing in the lower half-plane for $t-t_{0}>0$ ),

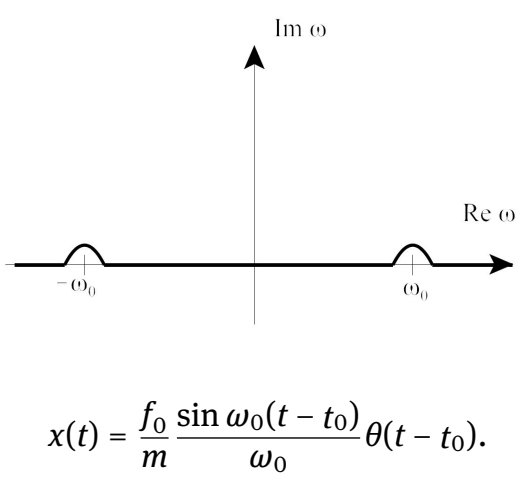


As a final example, we shall solve the equation

$$
\frac{d^{2} x}{d t^{2}}-\alpha^{2} x=f(t), \quad 0 \leqslant t<\infty
$$

subject to the boundary conditions $\frac{d x(0)}{d t}=b$ and $x(\infty)<\infty$, that is, $x(t)$ is bounded at infinity.

Since the range of the independent variable is restricted to $t \geqslant 0$, we can rule out Fourier transforms as a possible means of solution. However, that still leaves us with a choice between Fourier sine, Fourier cosine, and Laplace transforms. The fact that we have only one boundary condition at $t=0$ means that we lack an essential piece of information for use of the latter. Moreover, we are given $x(\infty)<\infty$ which is not needed for Laplace transforms but is needed for Fourier sine and cosine transforms. To decide between these final two options, we note that sine transforms require a knowledge of $x(0)$ while cosine transforms make use of $\frac{d x(0)}{d t}$. Thus, the clear choice of method in this case is to apply Fourier cosine transforms.

Since $\mathcal{F}_{C}\left\{x^{\prime \prime}(t)\right\}=-\sqrt{\frac{2}{\pi}} x^{\prime}(0)-\omega^{2} X_{C}(\omega)$ where $X_{C}(\omega)=\mathcal{F}_{C}\{x(t)\}$, the differential equation transforms to the subsidiary equation

$$
-\sqrt{\frac{2}{\pi}} b-\omega^{2} X_{C}(\omega)-\alpha^{2} X_{C}(\omega)=F_{C}(\omega), F_{C}(\omega)=\mathcal{F}_{C}\{f(t)\}
$$

with solution

$$
X_{C}(\omega)=-\sqrt{\frac{2}{\pi}} \frac{b}{\omega^{2}+\alpha^{2}}-\frac{F_{C}(\omega)}{\omega^{2}+\alpha^{2}} .
$$

The inverse transform of $\frac{1}{\omega^{2}+\alpha^{2}}$ is

$$
x_{1}(t)=\sqrt{\frac{2}{\pi}} \int_{0}^{\infty} \frac{\cos \omega t}{\omega^{2}+\alpha^{2}} d \omega=\frac{1}{\sqrt{2 \pi}} \int_{-\infty}^{\infty} \frac{\cos \omega t}{\omega^{2}+\alpha^{2}} d \omega .
$$

Since $\frac{1}{\omega^{2}+\alpha^{2}}$ is a rational function with a denominator of degree 2 and numerator of degree 0 , we can evaluate this integral by using a standard formula of residue calculus where, because $t \geqslant 0$, we select the version that sums over singularities in the upper half-plane:

$$
x_{1}(t)=-\left.2 \pi \frac{1}{\sqrt{2 \pi}} \operatorname{Im} \operatorname{Res}\left\{\frac{e^{i \omega t}}{\omega^{2}+\alpha^{2}}\right\}\right|_{\omega=i \alpha}=-\sqrt{2 \pi} \operatorname{Im}\left[\frac{e^{-\alpha t}}{2 \alpha i}\right]=\sqrt{\frac{\pi}{2}} \frac{e^{-\alpha t}}{\alpha} .
$$

This determines the inverse of the first term in our expression for $X_{C}(\omega)$ as well as of the factor multiplying $F_{C}(\omega)$ in the second term. Thus, all we need to complete the solution is to invoke the convolution theorem for Fourier cosine transforms. Thus,

$$
x(t)=-\frac{b}{\alpha} e^{-\alpha t}-\frac{1}{\sqrt{2 \pi}} \sqrt{\frac{\pi}{2}} \frac{1}{\alpha} \int_{0}^{\infty} f(\tau)\left[e^{-\alpha|t-\tau|}+e^{-\alpha(t+\tau)}\right] d \tau
$$




$$
=-\frac{b}{\alpha} e^{-\alpha t}-\frac{1}{2 \alpha} \int_{0}^{\infty} f(\tau)\left[e^{-\alpha|t-\tau|}+e^{-\alpha(t+\tau)}\right] d \tau
$$

where we have used the fact that $e^{-\alpha|t|}$ is the symmetric extension of $e^{-\alpha t}$.

That completes the solution of the problem. 